\title{
Mode II dominant fracture of layered composite beams and wide-plates: a homogenized structural approach
}

\author{
Roberta Massabò* and Hossein Darban
}

\begin{abstract}
Department of Civil, Chemical and Environmental Engineering, University of Genova, Via Montallegro 1, 16145, Genoa, Italy

*Corresponding author: mailto:roberta.massabo@unige.it
\end{abstract}

\begin{abstract}
Brittle delamination fracture under Mode II dominant conditions in unidirectional composite and layered beams and wide-plates is studied using a homogenized structural model based on a zigzag approach. The model captures the unstable propagation of cracks, snap-back and -through instabilities, the effects of the interaction of multiple cracks on the macrostructural response and of the layered structure on the energy release rate. The layered structure and the delaminations are described by introducing local enrichments, in the form of zigzag functions and cohesive interfaces, to a classical first-order shear deformation plate theory. The model applies to layers with principal material directions parallel to the geometrical axes, depends on only three displacement variables and the solution of specific problems requires only in-plane discretization, for any numbers of layers and delaminations. Closed form solutions are derived for the energy release rate in bi-material beams and applications are presented to homogeneous, bi-material and layered, simply supported and cantilever, bend-beams, with one and two delaminations.
\end{abstract}

Keywords: composites; zigzag theories; brittle fracture; delamination; J-integral

\begin{tabular}{|l|l|}
\hline Nomenclature & \\
\hline$a, a_{U}, a_{L}$ & Crack length, upper crack length, lower crack length \\
\hline $\bar{A}, \bar{B}, \bar{C}, \bar{D}, \bar{E}$ & Coefficients in the general solution \\
\hline$A_{22}, B_{22}, D_{22}, A_{44}$ & Stiffness coefficients in the constitutive equations of the homogenized beam \\
\hline$c$ & Length of ligament ahead of the crack tip \\
\hline$C^{r S}, C^{S 2}, C_{44}^{P}, C^{S}$ & Coefficients in the beam homogenized constitutive equations \\
\hline${ }^{(k)} E_{2},{ }^{(k)} G_{23}$ & Young modulus and shear modulus of layer $k=1, \ldots, n$ \\
\hline
\end{tabular}




\begin{tabular}{|c|c|}
\hline${ }^{(k)} \bar{E}_{2}$ & $\begin{array}{l}\text { Reduced Young's modulus in layer } k:{ }^{(k)} \bar{E}_{2}={ }^{(k)} E_{2} /{ }^{(k)}\left(1-v_{12} v_{21}\right) \text { for wide-plate; } \\
{ }^{(k)} \bar{E}_{2}={ }^{(k)} E_{2} \text { for beam; } \quad v_{12}, v_{21} \text { Poisson coefficients }\end{array}$ \\
\hline$G_{I I}$ & Mode II energy release rate \\
\hline$G_{I I C}$ & Mode II fracture energy \\
\hline$f_{3}$ & Transverse surface load \\
\hline${ }^{(k)} h$ & Thickness of layer $k=1, \ldots, n$ \\
\hline$J$ & J-integral \\
\hline$K_{S}^{k}$ & Stiffness of interface $k$ \\
\hline$k_{44}$ & Shear correction factor in the homogenized constitutive equations, $k_{44}=5 / 6$. \\
\hline${ }^{(i)} k_{44}$ & $\begin{array}{l}\text { Local shear correction factors relating generalized shear strain and sub-resultants } \\
\text { in the delaminated and intact arms of the bi-material beam. }\end{array}$ \\
\hline$N_{22}, M_{22}^{b}, Q_{2 g}$ & $\begin{array}{l}\text { Normal force, bending moment and generalized transverse shear force per unit } \\
\text { width, with } M_{22,2}^{b}\left(x_{2}\right)-Q_{2 g}\left(x_{2}\right)=0\end{array}$ \\
\hline${ }^{(i)} N_{22},{ }^{(i)} M_{22}^{b},{ }^{(i)} Q_{2 g}$ & $\begin{array}{l}\text { Force and moment sub-resultants per unit width in the delaminated and intact } \\
\text { arms of the bi-material beam. }\end{array}$ \\
\hline$P_{c r}$ & Critical load per unit width for crack propagation in the bend-beams. \\
\hline$Q_{2}^{b}$ & Transverse shear force \\
\hline$Q_{2}^{z}, M_{22}^{z S}, \hat{\sigma}_{2}$ & $\begin{array}{l}\text { Enrichment terms which define the generalized transverse shear force } \\
Q_{2 g}=Q_{2}^{b}+Q_{2}^{z}-M_{22}^{z S}-\hat{\sigma}_{2}\end{array}$ \\
\hline${ }^{(k)} v_{2},{ }^{(k)} v_{3}$ & Displacement components in layer $k$ \\
\hline$\hat{v}_{2}^{k}$ & Relative sliding displacement at the interface $k$ between layers $k$ and $k+1$ \\
\hline$v_{02}, \varphi_{2}, w_{0}$ & $\begin{array}{l}\text { Global variables: in-plane displacement, bending rotation and transverse } \\
\text { displacement (of reference surface when in first layer) }\end{array}$ \\
\hline $2^{(i)} \varepsilon_{23 g}$ & $\begin{array}{l}\text { Generalized shear strain energetically associated to the generalized sub-resultant, } \\
{ }^{(i)} Q_{2 g} \text {, in the intact and delaminated layers of bi-material beam. }\end{array}$ \\
\hline${ }^{(1)} \varphi_{2},{ }^{(2)} \varphi_{2}$ & Local rotations in the two layers of the bi-material beam \\
\hline$\gamma=\varphi_{2}+w_{0}, 2$ & $\begin{array}{l}\text { Global variable used to simplify solution and description of local fields; defines } \\
\text { shear strain in layer } k=1\end{array}$ \\
\hline$\Omega_{2}^{k}, \Lambda_{22}^{k}, \Psi_{22}^{k}, R_{S 22}^{k}$ & Coefficients accounting for the local enrichments \\
\hline$\prod$ & Total potential energy \\
\hline$\hat{\sigma}_{S}^{k}$ & Interfacial cohesive tractions (tangential) \\
\hline${ }^{(k)} \sigma_{i j},{ }^{(k)} \varepsilon_{i j}$ & Stress and strain components in the layer $k=1, . ., n$ \\
\hline
\end{tabular}




\begin{tabular}{|l|l|}
\hline${ }^{(k)} \sigma_{23}^{\text {post }}, 2^{(k)} \varepsilon_{23}^{\text {post }}$ & Transverse shear stress and strain calculated a posteriori from local equilibrium \\
\hline$\psi$ & Mode mixity phase angle, $\psi=\tan ^{-1}\left(\sqrt{G_{I I}} / \sqrt{G_{I}}\right)$ \\
\hline
\end{tabular}

\section{Introduction}

Layered composite materials are extensively being used for construction of structural components, in the form of beams, plates and shells, in various engineering applications. Sandwich panels for buildings or ship bulkheads, masts, decks and hulls, rudders and ailerons for aircrafts and wind turbine blades are just a few examples. Laminates and sandwiches are made with a large combination of materials in and within the layers, which include fiber reinforced polymer, ceramic or metallic matrices, wood, concrete, steel, polymeric and metallic foams, and glass. The layered structure is obtained through classical lamination techniques or using structural adhesives to join the layers. These components may be subjected to impacts and dynamic or repeated loadings, and suffer damage at several scales (damage in the fibers and the matrix, delamination and debonds at the interfaces between layers, failure of bonded/bolted attachments and global collapses). Knowing the extent of damage, through inspection methods and theoretical modeling, is critical to define the residual life and for damage tolerance assessments.

Modeling the evolution of delamination fracture in layered structures requires proper representation of various aspects. Delamination fracture is a discrete and localized damage event, which typically occurs simultaneously at the interface between different layers (multiple delamination) and may often be catastrophic. Delaminations may grow from manufacturing flaws or defects or may form, during service, due to impacts and the presence of through-thickness stresses. Delamination fracture may be brittle or controlled by cohesive mechanisms, due for instance to the bridging action developed by a through-thickness reinforcement or by cross-over fibers when the cracks meander through different layers. Delamination cracks are typically constrained to propagate along pre-defined paths at or near the interface between layers; however, for instance in sandwich systems with foam cores, the cracks may kink and dive into the core, a behavior which is controlled by the fracture toughness of the different components and the mode mixity conditions.

The most common numerical technique to analyze delamination fracture in layered structures uses the Finite Element Method and cohesive interface elements [1-7]. Damage within the layers may be accounted for using continuum damage approaches which modify the elastic constants of the layers when damage progresses. Three-dimensional finite elements are commonly used to study local 
behaviors, e.g. delaminations in joints, while beam, plate and shell elements are used to describe oneor two-dimensional structures. Discrete-layer cohesive-crack models, layer-wise and semi-layerwise theories are also used, often coupled with analytical solution methods or numerical techniques others than the finite element method [8-15]. Another common approach to study delamination fracture within finite element models uses the Virtual Crack Closure Technique [16] and requires remeshing techniques to follow delamination evolution.

One of the drawbacks of the classical approaches is the fine discretization which is necessary in the through-thickness direction to predict and model onset and growth of delaminations at the different interfaces. This complicates the finite element discretization and increases computational cost, especially since the problem is nonlinear. In addition, the requirement of a fine discretization limits the analytical and semi-analytical solutions to systems with a small number of layers/delaminations and simple loading and boundary conditions. The latter limitation should not be underestimated, since, as it will be shown later in the paper, analytical solutions yield an insight that could not be obtained through purely numerical calculations.

In this paper we investigate the use of a homogenized approach which removes the through thickness discretization of the problem, to model delamination fracture in layered structures. The model uses concepts developed in the original zigzag theory in $[17,18]$ to homogenize the problem and the multiscale strategy formulated in $[19,20]$ to account for the presence of delaminations by introducing cohesive interfaces, which are then included in the homogenization. The unknowns of the problem are substantially reduced, are independent of the numbers of layers or interfaces and coincide with those of a classical first-order shear deformation theory.

The basic assumption of the original zigzag theories for fully bonded structures is to enrich the displacement field of an Equivalent Single Layer theory, such as first- or higher-order shear deformation plate theory, by adding a local piecewise linear displacement field through so called zigzag functions [21,22]. The zigzag functions are then derived in terms of the global displacement variables through the imposition of continuity conditions at the layer interfaces. This reduces the number of unknowns of the problem and makes it independent of the number of layers. Most of the original zigzag theories, including that in [17] which is used in this paper, requires $C^{1}$ continuity for the displacement field, which complicates the finite element implementation; the purely displacement-based theories also require a-posteriori transverse shear stress recovery through the imposition of local equilibrium, which is generally not very accurate in a FE framework; in addition, it was initially thought that the original theories do not satisfy equilibrium of global forces at clamped boundaries (this is incorrect, see Sect. 2.2 for a discussion). New refined theories were later developed to overcome some of the drawbacks of the original theories [23-28]. 
The multiscale strategy formulated in [19,20] uses the zigzag theory in [17] and, in addition to introducing zigzag functions, enriches the global displacement field by introducing a throughthickness discontinuous local field which describes the presence of cohesive interfaces. The cohesive interfaces, which are characterized by piecewise linear cohesive tractions laws, are then included into the homogenization. Other authors have previously attempted to include imperfect interfaces into a zigzag formulation of the problem, however the models were not energetically consistent (see discussion in [20]). The approach developed in [19] has been successfully applied to analyze thermomechanical and wave propagation problems in wide-plates with imperfect or fully debonded interfaces proving the capabilities of the approach to accurately define global and local fields also in thick plates with highly inhomogeneous layups [29,30]. The model should be applicable to describe problems where fully debonded interfaces are present only in finite size regions, in order to simulate the presence of delaminations, and analyze their evolution. However, apart from a preliminary attempt in [31], this has not yet been proven and is the main objective of the work in this paper.

In [23] [32-36] different strategies have been applied to model the presence of mode II delaminations within a zigzag description of the problem. They are represented as thin and very compliant layers which are added to the regular layers of the system (compliant layer concept). Finite elements have been formulated which are able to capture the relative displacements between consecutive layers. They have also been used to simulate delamination damage progression and fracture by degrading the properties of the thin layers, using for instance a continuum damage approach. As recently noted in [37,38], however, the models which use this strategy and are based on the refined zigzag theories in $[25,26]$ have some limitations in plates with in-plane discontinuities in the material properties and yield inconsistent and unacceptable results, also for the global variables of the system, in the presence of delaminations.

The work presented here differs considerably from the previously published work. The delaminations are modelled using cohesive interfaces, which are then included into the homogenization, and fracture mechanics methods are applied to rigorously analyze and propagate cracks. The delamination cracks are then described as it is customary in classical-discrete layer cohesive-interface models. This description of delamination damage allows the use of classical zigzag theories [17], since it avoids the introduction of the compliant layers used in the above mentioned continuum damage approaches. In addition, the solutions presented here are analytical or semianalytical and this highlights the effects of the homogenization on the fracture parameters and limitations and advantages of the approach. The formulation of finite elements based on the homogenized model used in this paper is possible, but not carried out here, using approaches already used in the literature. 
The work is limited to mode II dominant problems in beams and wide-plates deforming in cylindrical bending and perfectly brittle fracture of the layers. The effects of the homogenization on the local fields in the layers and at the crack tip are explained using a bi-material beam with a single delamination as model system. A closed form solution is derived for the energy release rate in terms of crack tip force and moment sub-resultants which can be directly compared with accurate 2D solutions from the literature. Other derivation methods are also used and applied to various layered beams with single and multiple delaminations to verify the capabilities of the method.

The formulation and solution of the homogenized structural model is presented in Sects. 2.1 and 2.2. In Sect. 3. the energy release rate is calculated using different methods and a closed form solution in derived for homogeneous and bi-material beams. In Sect. 4, the model is applied to the solution of various bend-beams: homogeneous, bi-material, sandwich, simply supported and cantilever, with single and multiple delaminations; and the accuracy is verified through comparison with 2D solutions, discrete-layer models and experiments from the literature.

\section{Homogenized structural model for Mode II dominant fracture problems}

In this work we exploit the capabilities of the homogenized structural model formulated in [19] for beams and wide plates with continuous imperfect interfaces, to solve mode II dominant fracture problems and analyze delamination growth in layered structures, Fig. 1.a. Mode II dominant is used here to describe shear dominant mixed mode states where mode II deformation predominates and delamination growth can be assumed to be driven by mode II loading [39-41]. Typically, a crack may be considered to be in mode II dominant conditions when the phase angle, $\psi=\tan ^{-1}\left(\sqrt{G_{I I}} / \sqrt{G_{I}}\right)$ , which is a measure of Mode II to Mode I loading, is greater than $75^{\circ}$, with $\psi=90^{\circ}, 0^{\circ}$ the phase angles for pure Mode II and Mode I.

The multiscale formulation in [19] couples two mechanical models which are typically used for layered structures. The first is an Equivalent Single Layer (ESL) theory, namely first-order shear deformation or first-order shear and normal deformation plate theory $[42,43]$, and is used at the global scale (coarse grained model). The theories depend on a limited number of unknowns, accurately predict global fields but cannot reproduce the complex local fields which arise due to the layered structure, e.g. zigzag displacement fields, and the relative displacements occurring at the layer interfaces in the presence of delaminations. A discrete-layer cohesive interface model [8],[12] is then used at the local scale, which accounts for the layered structure and describes the imperfect interfaces and delaminations using piece-wise linear cohesive traction laws able to approximate the interfacial 
mechanisms, e.g. Figs. 1.b,c for Mode II problems. The two models are coupled by assuming a smallscale displacement field where the local variables enrich the field of the global theory. Homogenization is then applied to define the macro-displacement fields; and homogenized equilibrium equations are derived using a variational technique. They depend on the global variables of the model and on a set of coefficients which can be easily calculated, a priori, for fixed geometrical and material properties.

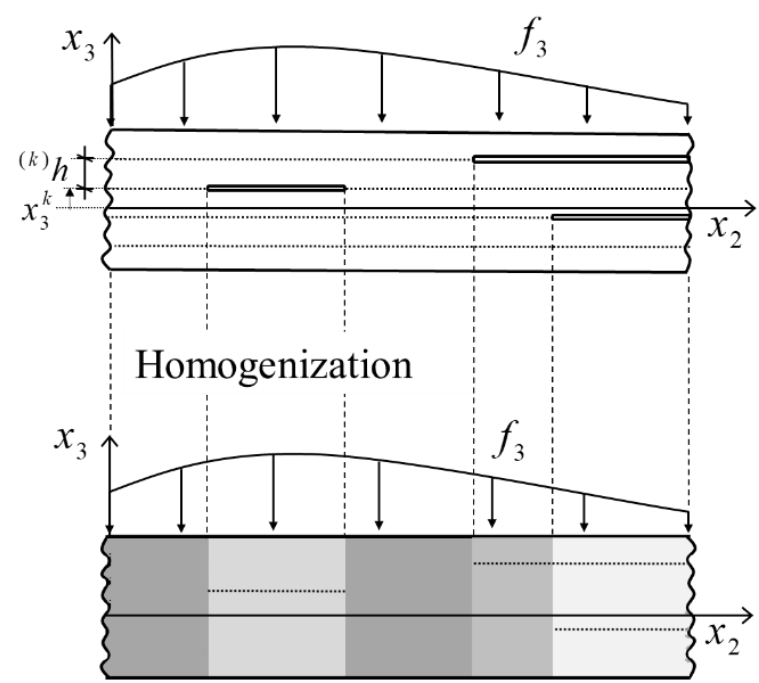

(a)
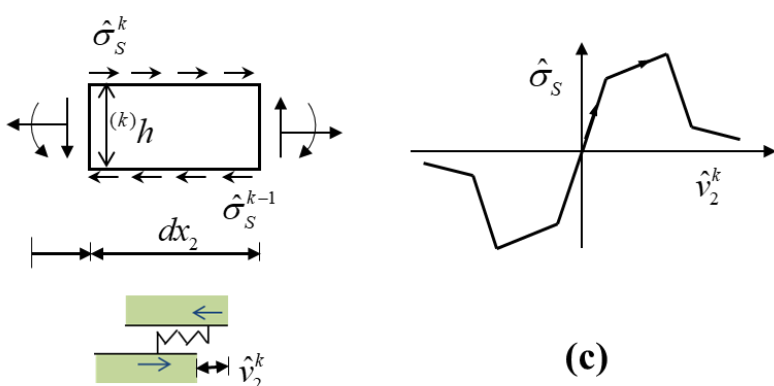

(c)

(b)

Fig. 1. (a) Layered beam/wide-plate with $n$ layers and delaminations under mode II dominant loading and homogenized description. (b) Element from a generic layer $k$ of the actual plate in (a) with interfacial cohesive tractions. (c) Piece-wise linear cohesive traction law relating tangential cohesive tractions and sliding displacement.

The equations of the homogenized model in [19] are recalled and used in this section to describe mode II dominant fracture problems where the interfaces are assumed to be rigid against mode I relative displacements, Fig. 1. It will be shown later that this assumption is analogous to the assumption of constrained-contact often used in discrete-layer descriptions of the problem. General solutions are derived for beams with arbitrary numbers of layers and delaminations and the local fields calculated a posteriori are discussed for the exemplary case of a cracked bi-material beam.

\subsection{Model assumptions and homogenization}

The $n$ layers of the beam/wide-plate in Fig. 1.a are linearly elastic, homogenous and orthotropic with principal material axes parallel to the geometrical axes, $x_{1}-x_{2}-x_{3}$. The layer $k$, with $k=1$ the lower 
layer, is defined by the coordinates of its lower and upper surfaces, $x_{3}^{k-1}$ and $x_{3}^{k}$, and has thickness ${ }^{(k)} h$ (the superscript $(k)$ on the left of a quantity shows association with the layer $k$; the superscript $k$ on the right defines the interface between layers). The layers are assumed to be incompressible in the thickness direction and the transverse normal stresses ${ }^{(k)} \sigma_{33}$ to be negligible compared to the other stress components. Under these assumptions and if the wide-plate deforms in cylindrical bending, the nonzero displacement components are ${ }^{(k)} v_{2}={ }^{(k)} v_{2}\left(x_{2}, x_{3}\right)$ and ${ }^{(k)} v_{3}=w_{0}\left(x_{2}\right)$, and the relevant constitutive and compatibility equations of the layer $k$ are:

$$
\begin{aligned}
& { }^{(k)} \sigma_{22}={ }^{(k)} \bar{E}_{2}{ }^{(k)} \varepsilon_{22} \quad ; \quad{ }^{(k)} \sigma_{23}={ }^{(k)} G_{23} 2^{(k)} \varepsilon_{23} \\
& { }^{(k)} \varepsilon_{22}\left(x_{2}, x_{3}\right)={ }^{(k)} v_{2},,_{2}\left(x_{2}, x_{3}\right) \quad ; \quad 2^{(k)} \varepsilon_{23}\left(x_{2}, x_{3}\right)={ }^{(k)} v_{2},{ }_{3}\left(x_{2}, x_{3}\right)+{ }^{(k)} v_{3,2}\left(x_{2}, x_{3}\right)
\end{aligned}
$$

where ${ }^{(k)} \sigma_{i j}$ and ${ }^{(k)} \varepsilon_{i j}$ for $i, j=2,3$ are stress and infinitesimal strain components; ${ }^{(k)} \bar{E}_{2}={ }^{(k)} E_{2} /{ }^{(k)}\left(1-v_{12} v_{21}\right)$ for a wide-plate and ${ }^{(k)} \bar{E}_{2}={ }^{(k)} E_{2}$ for a beam, with ${ }^{(k)} E_{2},{ }^{(k)} G_{23},{ }^{(k)} v_{12}$ and ${ }^{(k)} v_{21}$ relevant Young's modulus, shear modulus and Poisson coefficients of the layer $k$ (a comma followed by a subscript denotes a derivative with respect to the corresponding coordinate). Cohesive interfaces are introduced between the layers in the schematic in Fig. 1.a. The $(n-1)$ interfaces are zero-thickness mathematical surfaces where material properties and displacements are discontinuous while surface tractions are continuous. The mechanical behavior of the interfaces is governed by a cohesive traction law which relates the relative sliding displacement of the layers at the interface:

$$
\hat{v}_{2}^{k}\left(x_{2}\right)={ }^{(k+1)} v_{2}\left(x_{2}, x_{3}=x_{3}^{k}\right)-{ }^{(k)} v_{2}\left(x_{2}, x_{3}=x_{3}^{k}\right)
$$

to the interfacial cohesive tractions, $\hat{\sigma}_{S}^{k}\left(x_{2}, x_{3}=x_{3}^{k}\right)$ Fig. 1.c. In order to approximate perfectly brittle fracture, the cohesive traction law is assumed to be linear, with a very stiff initial branch, up to a critical sliding displacement $\hat{v}_{2 c}$, beyond which the cohesive tractions vanish [1] [12]:

$$
\hat{\sigma}_{S}^{k}\left(x_{2}, x_{3}=x_{3}^{k}\right)=\left\{\begin{array}{cc}
K_{S}^{k} \hat{v}_{2}^{k}\left(x_{2}\right) & \text { for } \hat{v}_{2}^{k}\left(x_{2}\right) \leq \hat{v}_{2 c} \\
0 & \text { for } \hat{v}_{2}^{k}\left(x_{2}\right) \geq \hat{v}_{2 c}
\end{array}\right.
$$


The coordinates where $\hat{v}_{2}^{k}=\hat{v}_{2 c}$ define the crack tips, Fig. 2.a. To simplify the treatment of the problem, the cohesive laws are approximated by the following two-branch law, Fig. 2.b:

$$
\hat{\sigma}_{S}^{k}\left(x_{2}, x_{3}=x_{3}^{k}\right)= \begin{cases}K_{S}^{k} \hat{v}_{2}^{k}\left(x_{2}\right) & \text { for } \hat{v}_{2}^{k}\left(x_{2}\right) \leq \hat{v}_{2 c} \text { with } 1 / K_{S}^{k} \rightarrow 0 \\ K_{S}^{k} \hat{v}_{2}^{k}\left(x_{2}\right) & \text { for } \hat{v}_{2}^{k}\left(x_{2}\right) \geq \hat{v}_{2 c} \quad \text { with } K_{S}^{k} \rightarrow 0\end{cases}
$$

where the interfacial shear tractions are assumed to be proportional to the relative displacement. The advantage of the law in Eq. (4) is that it allows to formulate and solve the problem for generic linear traction laws with stiffness $K_{S}^{k}, k=1, \ldots, n-1$, and then use the solution to describe the different portions of the beam. In the numerical solutions presented in Sect. 4 the numerical values of the interfacial stiffnesses in the intact/delaminated portions will be chosen as large/small as possible to avoid numerical problems; in the closed form solutions, a perturbation technique will be used and zero order expansions of the variables will describe the fully-bonded and delaminated regions.

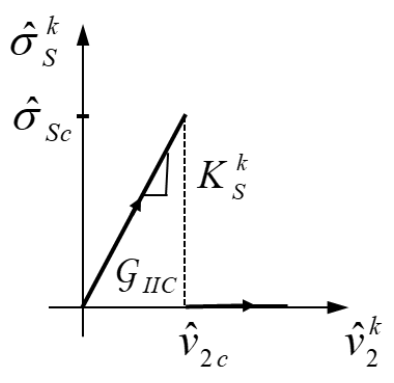

(a)

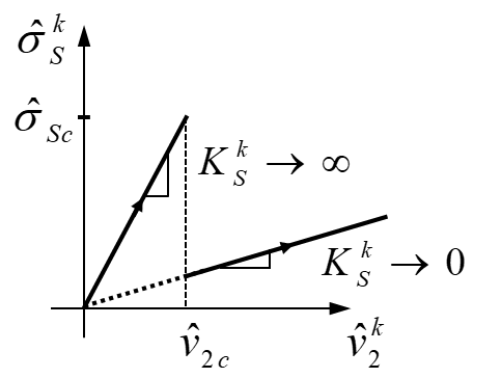

(b)

Fig. 2. (a) Interfacial traction law to approximate mode II Linear Elastic Fracture Mechanics. (b) Interfacial traction law used in the solution of the homogenized model.

Following [17-19], a small-scale displacement field is assumed for the layer $k$ :

$$
\begin{aligned}
& { }^{(k)} v_{2}\left(x_{2}, x_{3}\right)=v_{02}\left(x_{2}\right)+x_{3} \varphi_{2}\left(x_{2}\right)+\sum_{i=1}^{k-1} \Omega_{2}^{i}\left(x_{2}\right)\left(x_{3}-x_{3}{ }^{i}\right)+\sum_{i=1}^{k-1} \hat{v}_{2}^{i}\left(x_{2}\right) \\
& { }^{(k)} v_{3}\left(x_{2}, x_{3}\right)=w_{0}\left(x_{2}\right)+x_{3} \varphi_{3}\left(x_{2}\right)
\end{aligned}
$$

where local terms are introduced to enrich the global field of the first order theory: zigzag functions, $\Omega_{2}^{i}\left(x_{2}\right)\left(x_{3}-x_{3}^{i}\right)$, and relative sliding displacements at the layer interfaces, $\hat{v}_{2}^{i}\left(x_{2}\right)$. The global variables are the in-plane displacement, $v_{02}\left(x_{2}\right)$, the bending rotation, $\varphi_{2}\left(x_{2}\right)$ and the transverse 
displacement $w_{0}\left(x_{2}\right)$; they directly define the generalized displacements of the reference surface when this falls into the first layer. The small scale displacements in the plate are then defined by a total of three global variables and $2(n-1)$ local variables.

The zigzag functions are derived as functions of the global variables by imposing continuity of the shear tractions of the layers at the interfaces; this is done by posing ${ }^{(k+1)} \sigma_{23}\left(x_{3}=x_{3}^{k}\right)={ }^{(k)} \sigma_{23}\left(x_{3}=x_{3}^{k}\right)$ for $k=1, \ldots,(n-1)$ and using Eqs. (1). The relative sliding displacements at the interfaces are derived as function of the global variables by equating the interfacial cohesive tractions, Eq. (4), to the shear tractions, using Eqs. (1) [19]. This yields:

$$
\Omega_{2}^{k}=\left(w_{0,2}+\varphi_{2}\right) \Lambda_{22}^{k} \quad ; \quad \hat{v}_{2}^{k}=\left(w_{0,2}+\varphi_{2}\right) \Psi_{22}^{k}
$$

with

$$
\Lambda_{22}^{k}={ }^{(1)} G_{23}\left(\frac{1}{{ }^{(k+1)} G_{23}}-\frac{1}{{ }^{(k)} G_{23}}\right) \quad ; \quad \Psi_{22}^{k}=\frac{{ }^{(k+1)} G_{23}}{K_{S}^{k}}\left(1+\sum_{j=1}^{k} \Lambda_{22}^{(j)}\right)
$$

Substituting the small-scale variables into Eq. (5) yields the macro-scale displacements in the layer $k$ :

$$
\begin{aligned}
& { }^{(k)} v_{2}\left(x_{2}, x_{3}\right)=v_{02}\left(x_{2}\right)+x_{3} \varphi_{2}\left(x_{2}\right)+\left[w_{0}, 2\right. \\
& \left.{ }^{(k)} v_{3}\left(x_{2}\right)+\varphi_{2}\right)=w_{0}\left(x_{2}\right)
\end{aligned}
$$

with

$$
R_{S 22}^{k}\left(x_{3}\right)=\sum_{i=1}^{k-1}\left[\Lambda_{22}^{i}\left(x_{3}-x_{3}^{i}\right)+\Psi_{22}^{i}\right]
$$

Strain and stress components, interfacial tractions and relative crack displacements are obtained in terms of the global variables by substituting Eq. (8) into Eqs. (1)(2)(4):

$$
\begin{aligned}
& { }^{(k)} \varepsilon_{22}\left(x_{2}, x_{3}\right)=v_{02,}\left(x_{2}\right)+x_{3} \varphi_{2},{ }_{2}\left(x_{2}\right)+\left[w_{0},_{22}\left(x_{2}\right)+\varphi_{2},,_{2}\left(x_{2}\right)\right] R_{S 22}^{k} \\
& 2^{(k)} \varepsilon_{23}\left(x_{2}, x_{3}\right)=\left[w_{0,2}\left(x_{2}\right)+\varphi_{2}\left(x_{2}\right)\right]\left(1+R_{S 22,3}^{k}\right)
\end{aligned}
$$




$$
\begin{aligned}
& { }^{(k)} \sigma_{22}\left(x_{2}, x_{3}\right)={ }^{(k)} \bar{E}_{2}\left(v_{02},{ }_{2}\left(x_{2}\right)+x_{3} \varphi_{2},{ }_{2}\left(x_{2}\right)+\left[w_{0},{ }_{22}\left(x_{2}\right)+\varphi_{2,2}\left(x_{2}\right)\right] R_{S 22}^{k}\right) \\
& { }^{(k)} \sigma_{23}\left(x_{2}\right)={ }^{(k)} G_{23}\left[w_{0}, 2\left(x_{2}\right)+\varphi_{2}\left(x_{2}\right)\right]\left(1+R_{S 22,3}^{k}\right) \\
& \hat{\sigma}_{S}^{k}={ }^{(k+1)} G_{23}\left(1+\sum_{j=1}^{k} \Lambda_{22}^{j}\right)\left[w_{0}, 2\left(x_{2}\right)+\varphi_{2}\left(x_{2}\right)\right] \\
& \hat{v}_{2}^{k}=\Psi_{22}^{k}\left[w_{0}, 2\left(x_{2}\right)+\varphi_{2}\left(x_{2}\right)\right]
\end{aligned}
$$

In a plate with layers having the same elastic properties, $\Lambda_{22}^{k}=0$ is zero since ${ }^{(k+1)} G_{23}={ }^{(k)} G_{23}$; in the intact portions of such plate, where $1 / K_{S}^{k}=\Psi_{22}^{k}=0$, also the relative crack displacements, $\hat{v}_{2}^{k}$, vanish and the displacement field coincides with that of the first order shear deformation theory [42]. If the elastic constants of the layers differ, $\Lambda_{22}^{k} \neq 0$ and in the intact portions of the plate the displacement field coincides with that assumed by the original zigzag theory in [17].

The transverse shear stresses in Eq. (10) are constant through the thickness, since ${ }^{(k)} G_{23}\left(1+R_{S 22}^{k}, 3\right)={ }^{(k+1)} G_{23}\left(1+R_{S 22,3}^{k+1}\right)$. This is a consequence of the a priori imposition of continuity of the shear tractions at the interface and the assumption of a first order global displacement field. To account for this limitation a shear correction factor will be introduced in the equilibrium equations of the problem (Sect. 2.2). In addition, in the delaminated portions of the plate, where the interfacial tractions vanish, which implies $w_{0,2}+\varphi_{2}=0$ after Eq. (10), both shear strains and stresses vanish. Following the approach which is commonly used for the structural low order theories, accurate predictions of the transverse shear stresses and strains, in both delaminated and intact regions, can be made a posteriori from the bending stresses by imposing local equilibrium:

$$
{ }^{(k)} \sigma_{22,2}+{ }^{(k)} \sigma_{23}^{\text {post }}, 3=0 \quad ; \quad 2^{(k)} \varepsilon_{23}^{\text {post }}=\frac{{ }^{(k)} \sigma_{23}^{\text {post }}}{{ }^{(k)} G_{23}}
$$

The use of the equations above allows to accurately describe the complex local fields which arise in multilayered plates with imperfect or fully bonded interfaces, also in very thick and highly anisotropic plates (see [19,29]). However, stress recovery using the equation above is not very accurate in a finite element framework [27]. Due to the vanishing of the shear strains in the delaminated regions of the plate, the transverse displacements in Eq. (8) will only account for the bending contributions and this may affect the load re-distribution between different regions in statically indeterminate systems.

\subsection{Constitutive and equilibrium equations and general solution}


The homogenized equilibrium equations and boundary conditions in the beam in Fig. 1.a, are derived using the Principle of Virtual Works [19]. The variationally consistent equilibrium equations and boundary conditions in terms of force and moment resultants per unit width, $N_{22}, M_{22}^{b}, Q_{2 g}$, and surface tractions, $f_{3}$, are:

$$
\begin{aligned}
& N_{22,,_{2}}\left(x_{2}\right)=0 \\
& M_{22,2}^{b}\left(x_{2}\right)-Q_{2 g}\left(x_{2}\right)=0 \\
& Q_{2 g}, 2\left(x_{2}\right)+f_{3}\left(x_{2}\right)=0
\end{aligned}
$$

and

$$
\begin{array}{lll}
N_{22} n_{2}=\tilde{N}_{2} & \text { or } & v_{02}=\tilde{v}_{02} \\
M_{22}^{b} n_{2}=\tilde{M}_{2}^{b} & \text { or } & \varphi_{2}=\tilde{\varphi}_{2} \\
Q_{2 g} n_{2}=\tilde{N}_{3} & \text { or } & w_{0}=\tilde{w}_{0} \\
M_{22}^{z S} n_{2}=\tilde{M}_{2}^{z S} & \text { or } & w_{0,2}=\tilde{w}_{0}, 2
\end{array}
$$

where the tilde defines prescribed values at the plate edges and $n_{2}= \pm 1$ is the component of the outward unit normal along $x_{2}$.

Force and moment resultants are given by:

$$
\begin{aligned}
& \left(N_{22}, M_{22}^{b}\right)=\sum_{k=1}^{n} \int_{x_{3}^{k-1}}^{x_{3}^{k}}{ }^{(k)} \sigma_{22}\left(1, x_{3}\right) d x_{3} \\
& Q_{2 g}\left(x_{2}\right)=Q_{2}^{b}\left(x_{2}\right)+Q_{2}^{z}\left(x_{2}\right)-M_{22}^{z S},{ }_{2}\left(x_{2}\right)-\hat{\sigma}_{2}\left(x_{2}\right)
\end{aligned}
$$

with

$$
\begin{aligned}
& Q_{2}^{b}=\sum_{k=1}^{n} \int_{x_{3}^{k-1}}^{x_{3}^{k}}{ }^{(k)} \sigma_{23} d x_{3} \quad ; \quad M_{22}^{z S}=\sum_{k=1}^{n} \int_{x_{3}^{k-1}}^{x_{3}^{k}}{ }^{(k)} \sigma_{22} R_{S 22}^{k} d x_{3} \\
& Q_{2}^{z}=\sum_{k=1}^{n} \int_{x_{3}^{k-1}}^{x^{k}}{ }^{(k)} \sigma_{23} R_{S 22}^{k},{ }_{3} d x_{3} \quad ; \quad \hat{\sigma}_{2}=-\sum_{k=1}^{n-1}{ }^{(k+1)} G_{23}\left(1+\sum_{j=1}^{k} \Lambda_{22}^{j}\right) \hat{v}_{2}^{k}
\end{aligned}
$$

The form of the equilibrium equations is analogous to that of the global first order shear deformation theory and the normal force and bending moments, $N_{22}$ and $M_{22}^{b}$, have the usual 
definitions. The effects of the local enrichments appear in the generalized transverse shear force, $Q_{2 g}$ which substitutes for the classical transverse shear force, $Q_{2}^{b}$, in the second and third equilibrium equation (12). As shown in the third equation in (12), the variationally consistent generalized transverse shear force is statically equivalent, at any arbitrary section of the plate with outward normal $\boldsymbol{n}=\{0,1,0\}^{\mathrm{T}}$, to the vertical equilibrant of the external forces acting on the portion of the plate to the right of the section. $Q_{2 g}$ therefore equates the resultant of the a posteriori calculated transverse shear stresses ${ }^{(k)} \sigma_{23}^{\text {post }}$, Eq. (11):

$$
Q_{2 g}=\sum_{k=1}^{n} \int_{x_{3}^{k-1}}^{x_{3}^{k}}{ }^{(k)} \sigma_{23}^{p o s t} d x_{3}
$$

The resultant of the a-posteriori calculated shear stresses is variationally consistent in this model.

In the intact portions of the plate where $\hat{v}_{2}^{k}=0$, which implies $\hat{\sigma}_{2}=0$ Eq. (15), the equilibrium equations coincide with those of the original first order zigzag theory in [17] for fully bonded plates. In the intact portion of a homogenous plate or when the zigzag effects are neglected by posing $\Lambda_{22}^{k}=0$, then $M_{22}^{z S}=Q_{2}^{z}=\hat{\sigma}_{2}=0$, the generalized shear force $Q_{2 g}$ equals the transverse shear force $Q_{2}^{b}$ and the equilibrium equations are those of first order shear deformation theory.

As a consequence of the homogenization, the boundary conditions, Eq. (13), are defined in terms of global quantities. This highlights two limitations of the model when applied to cracked bodies. First, it is not applicable to problems with edge cracks where sub-forces and sub-moments acts at the delaminated arms in opposite directions. For instance if two equal magnitude shear forces were applied at the two delaminated arm ends with opposite signs, the generalized shear force $Q_{2 g}$, which is the net value over the thickness, would be zero. However, these boundary conditions are essentially limited to laboratory test specimens and are unlikely to occur in practical cases, where the delamination arise between the internal layers and the loads are applied on the outer surfaces of the structure. The second limitation is the impossibility of the present model to satisfy local conditions in a boundary region at the crack tip cross-sections.

At clamped supports, where the boundary conditions Eq. (13) impose $\tilde{\varphi}_{2}=\tilde{w}_{0},_{2}=0$, the transverse shear strains and stresses in Eq. (10), vanish, which implies $Q_{2}^{b}=0$; this result does not affect the global solution of the problem, as it was erroneously thought in early applications of the original zigzag model to fully bonded plates [25], since global equilibrium is satisfied at the clamped 
edges by a nonzero generalized transverse shear force, $Q_{2 g}$, which is the variationally consistent relevant internal force. However, as for the crack tip cross sections, since the boundary conditions are in terms of global quantities, boundary regions are generated at the clamped edges where the local fields are not accurately predicted; the size of these regions depend on the mismatch of the elastic constants of the layers and on the stiffness of the interface and is very small in plates where the mismatch is small and the interfacial stiffness is very large or very small [31][38].

The constitutive equations of the layered beam are derived by substituting stresses and interfacial relative sliding displacement from Eqs. (10) into Eq. (14)[44]:

$$
\begin{aligned}
& \left\{\begin{array}{l}
N_{22} \\
M_{22}^{b} \\
M_{22}^{z S}
\end{array}\right\}=\left[\begin{array}{lll}
A_{22} & B_{22}+C^{0 S} & C^{0 S} \\
B_{22} & D_{22}+C^{1 S} & C^{1 S} \\
C^{0 S} & C^{1 S}+C^{S 2} & C^{S 2}
\end{array}\right]\left\{\begin{array}{c}
v_{02}, 2 \\
\varphi_{2,} \\
w_{0},{ }_{22}
\end{array}\right\}
\end{aligned}
$$

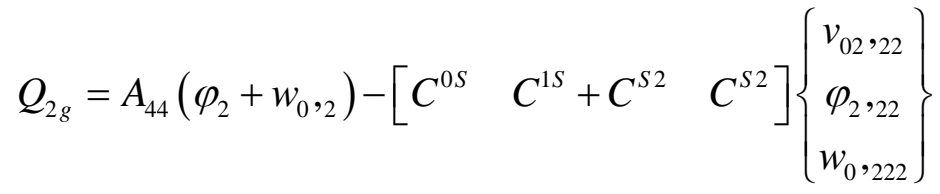

with:

$$
\begin{aligned}
& \left(A_{22}, B_{22}, \mathrm{D}_{22}\right)=\sum_{k=1}^{n}{ }^{(k)} \bar{E}_{2} \int_{x_{3}^{k-1}}^{x_{3}^{k}}\left(1, x_{3}, x_{3}{ }^{2}\right) d x_{3} \\
& A_{44}=k_{44} C_{44}^{P}+C^{S}
\end{aligned}
$$

where the coefficients, $C^{r S}, C^{S 2}, C_{44}^{P}$ and $C^{S}$ for $r=0,1,2$, depend on the geometry, the layup and the status of the interface and are defined in Eq. (47) in Appendix A. The coefficients with superscript $S$ depend on the local enrichments and they assume different values in the intact and delaminated regions; $M_{22}^{z S}$ is the moment resultant due to the local enrichments, Eq. (15). In a plate with fully bonded interfaces, with $1 / K_{S}^{k} \rightarrow 0$, and where the zig-zag contributions are zero or neglected by posing $\Lambda_{22}^{k}=0$, the constitutive equations coincide with those of First Order Shear Deformation theory, since $C^{r S}=C^{S 2}=C^{S}=0$. In this case the response is described by the classical extensional, coupling and bending stiffnesses, $A_{22}, \quad B_{22}, D_{22}$, and is the classical shear stiffness $A_{44}=k_{44} \sum_{k=1}^{n}{ }^{(k)} G_{23}{ }^{(k)} h$.

A shear correction factor, $k_{44}$, is introduced in the equations above in order to recover the constitutive equations of the equivalent first order shear deformation theory in the limiting case of a fully bonded homogeneous plate, for which $k_{44}=5 / 6$ under static loading. The correction factor 
required for homogeneous plates can be used also for layered plates since the zigzag effects are accounted for through the multiscale treatment; this was observed in [19] for static problems and in [12] for dynamic problems. In Eq. (17) the shear correction factor is introduced so that $2^{(1)} \varepsilon_{23}\left(x_{2}, x_{3}\right)=\left(Q_{2}^{b}+Q_{2}^{z}\right) /\left(k_{44} C_{44}^{p}\right)$.

The homogenized equilibrium equations are defined in terms of global displacements, by substituting the constitutive equations (17) into Eq. (12). The eight order system is:

$$
\begin{aligned}
& A_{22} v_{02,22}+\left(B_{22}+C^{0 S}\right) \varphi_{2,22}+C^{0 S} w_{0,222}=0 \\
& \left(B_{22}+C^{0 S}\right) v_{02,{ }_{22}}+\left(D_{22}+2 C^{1 S}+C^{S 2}\right) \varphi_{2},{ }_{22}+\left(C^{1 S}+C^{S 2}\right) w_{0},_{222}-A_{44}\left(w_{0},{ }_{2}+\varphi_{2}\right)=0 \\
& C^{0 S} v_{02},{ }_{222}+\left(C^{1 S}+C^{S 2}\right) \varphi_{2},{ }_{222}+C^{S 2} w_{0},{ }_{2222}-A_{44}\left(w_{0},{ }_{22}+\varphi_{2},{ }_{2}\right)-f_{3}=0
\end{aligned}
$$

In a beam with traction-free external surfaces, $f_{3}=0$, the general solution is [44]:

$$
\begin{aligned}
& w_{0}=\frac{\bar{D} \bar{B}+\bar{E}}{\bar{B} \sqrt{\bar{B}}}\left[c_{1} e^{\sqrt{\bar{B}} x_{2}}-c_{2} e^{-\sqrt{\bar{B}} x_{2}}\right]+\frac{\bar{E} c_{3}}{6}\left(x_{2}\right)^{3}+c_{4}\left(x_{2}\right)^{2}+c_{5} x_{2}+c_{6} \\
& \varphi_{2}=\left(1-\frac{\bar{D} \bar{B}+\bar{E}}{\bar{B}}\right)\left[c_{1} e^{\sqrt{\bar{B}} x_{2}}+c_{2} e^{-\sqrt{\bar{B}} x_{2}}\right]-\frac{\bar{E} c_{3}}{2}\left(x_{2}\right)^{2}-2 c_{4} x_{2}+c_{3}-c_{5} \\
& v_{02}=\frac{\bar{B}\left(B_{22} \bar{D}-B_{22}-C^{0 S}\right)+B_{22} \bar{E}}{A_{22} \bar{B}}\left[c_{1} e^{\sqrt{\bar{B}} x_{2}}+c_{2} e^{-\sqrt{\bar{B}} x_{2}}\right]+\frac{c_{3} B_{22} \bar{E}}{2 A_{22}}\left(x_{2}\right)^{2}+c_{7} x_{2}+c_{8}
\end{aligned}
$$

where $\bar{B}, \bar{C}, \bar{D}$ and $\bar{E}$ depend on the coefficients in Eq. (47) and are given in Eq. (49) and $c_{i}$ for $i$ $=1, \ldots, 8$, are integration constants. From Eq. (10) and (19), the transverse shear strain of the first layer, $\gamma=2^{(1)} \varepsilon_{23}=\varphi_{2}+w_{0}, 2$, which will be used in the following derivations is defined by:

$$
\gamma=2^{(1)} \varepsilon_{23}=\varphi_{2}+w_{0}, 2=c_{1} e^{\sqrt{\bar{B}} x_{2}}+c_{2} e^{-\sqrt{\bar{B}} x_{2}}+c_{3}
$$

The derivation constants in Eq. (19) necessitates the imposition of boundary conditions and continuity conditions on the global variables, $v_{02}, \varphi_{2}, w_{0}, w_{0,2}, N_{22}, M_{22}^{b}, Q_{2 g}$ and $M_{22}^{z S}$ at the joining cross sections. In the intact region of the plate, where $1 / K_{S}^{k} \rightarrow 0$, Eq. (19) holds with with $\Psi_{22}^{k}=0, \hat{v}_{2}^{k}=0, C_{22}^{S}=0$. In the delaminated region of the plate, for $K_{S}^{k} \rightarrow 0$, some of the coefficients in Eq. (19) remain finite $\bar{A} \rightarrow 1, A_{22}, B_{22}, D_{22}, \bar{E}, \bar{D} \bar{B}, C^{0 S} \bar{B}$, while others go to 
infinity with various orders [29]. Concepts from perturbation analysis will be used in the following to elaborate the solution in this limit.

\subsection{Local fields in an edge cracked element}

To clarify how the homogenized approach preserves the description of the local fields of classical discrete layer cohesive-zone models, the special case of a bi-material beam with a single delamination is examined and the local fields derived in closed form from the solution in Eq. (19). The fields will be discussed with reference to a cracked element of length $(a+c)$ extracted from the "actual" beam in Fig. 1.a and subjected only to moment and force resultants acting at the element ends, ${ }^{(i)} N,{ }^{(i)} Q,{ }^{(i)} M$ , with $\mathrm{i}=0,1,2$ for the intact, delaminated and substrate arms, Fig. 3.a. It will be shown that, at sufficient distance from the crack tip, the local fields calculated a posteriori coincide with those of a classical discrete-layer model which uses Timoshenko beam theory to describe the layers.

Figure 3.b shows the "homogenized" crack tip element and the global force and moment resultants, Eqs. (14), acting at $x_{2}=-a, N_{22}, Q_{2 g}, M_{22}^{b}$, and at $x_{2}=c,{ }^{(0)} N_{22},{ }^{(0)} Q_{2 g},{ }^{(0)} M_{22}^{b}$. The origin of the global system $x_{1}-x_{2}-x_{3}$ is at the crack tip cross section and $x_{2}$ is along the neutral axis of the intact portion of the element (to simplify the derivation it is assumed that the neutral axis falls into the lower layer); local coordinates are introduced at the mid-thickness of the first, ${ }^{(1)} X_{3}=x_{3}+{ }^{(1)} e$, and second layer, ${ }^{(2)} X_{3}=x_{3}-{ }^{(2)} e$, with ${ }^{(1)} e$ and ${ }^{(2)} e$ the distances between the reference plane $x_{3}=0$ and the geometrical mid-thickness of the layers (Fig. 3.c).

A schematic of the longitudinal displacements in the layers of the delaminated portion, ${ }^{(1)} v_{2}$ and ${ }^{(2)} v_{2}$, after Eq. (8) is shown in Fig. 3.c. The derivatives, ${ }^{(1)} v_{2},{ }_{3}$ and ${ }^{(2)} v_{2},_{3}$, define the slopes which represent the local rotations of the layers, ${ }^{(i)} \varphi_{2}={ }^{(i)} v_{2},_{3}$ :

$$
\begin{aligned}
& { }^{(1)} \varphi_{2}\left(x_{2}\right)=\varphi_{2}\left(x_{2}\right) \\
& { }^{(2)} \varphi_{2}\left(x_{2}\right)=\varphi_{2}\left(x_{2}\right)+\left[\varphi_{2}\left(x_{2}\right)+w_{0}\left(x_{2}\right),{ }_{2}\right] \Lambda_{22}^{1}
\end{aligned}
$$

In a homogeneous element, $\Lambda_{22}^{1}=0$ and the local rotations coincide with the global rotation, ${ }^{(1)} \varphi_{2}={ }^{(2)} \varphi_{2}=\varphi_{2}$, everywhere in the element. A similar conclusion holds in the delaminated region in a bi-material element. This is proven by applying concepts of perturbation analysis to the shear deformation of the first layer, $\gamma\left(x_{2}\right)$ in Eq. (20), and to the relative crack displacement, $\hat{v}_{2}^{1}\left(x_{2}\right)$, Eq. 
(10). By assuming $K_{s}^{1} \rightarrow 0$, the functions $\gamma\left(x_{2}\right)$ and $\hat{v}_{2}^{1}\left(x_{2}\right)$ are expanded into power series of $K_{s}^{1}$

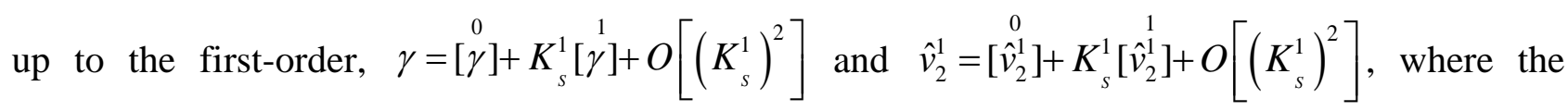
superscript $\left[\stackrel{i}{\bullet}\right.$ ] indicates the order of the expansion term. Since $\hat{v}_{2}^{1}={ }^{(2)} G_{23} \gamma\left(x_{2}\right)\left(1+\Lambda_{22}^{(1,1)}\right)\left(K_{S}^{1}\right)^{-1}$, after Eqs. (7),(10), and its zero order expansion, $\left[\hat{v}_{2}^{1}\right]$, must be finite in the delaminated region, then the zero order expansion of $\gamma$ must vanish, $\left[\begin{array}{c}{[\gamma]} \\ {[}\end{array}=0\right.$. As a consequence, the dominant terms of the local rotations of the layers in Eq. (21) coincide with the global rotation, ${ }^{(1)} \varphi_{2}={ }^{(2)} \varphi_{2}=\varphi_{2}$.

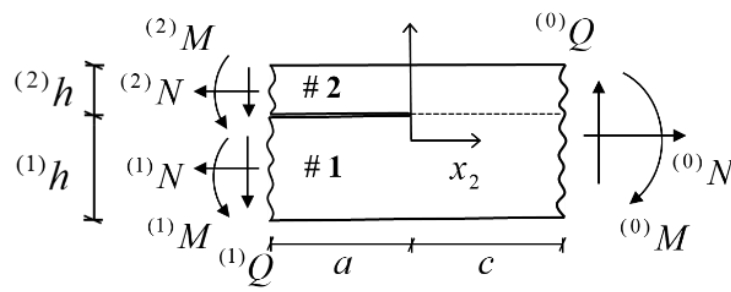

(a)
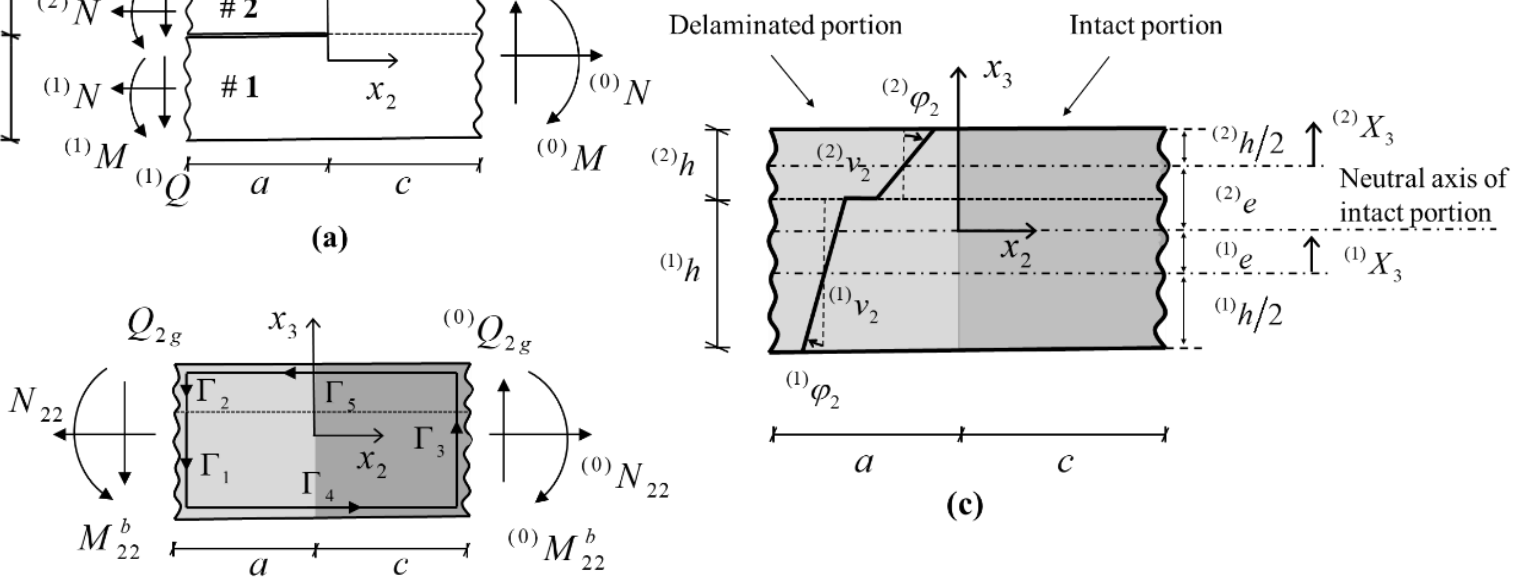

(c)

(b)

Fig. 3. (a) Schematic of crack tip element in a delaminated bi-material beam with one delamination and no surface loads showing force and moment resultants acting at the end cross sections. (b) Homogenized crack tip element with global force and moment resultants and J-integral path. (c) Local coordinates and rotations in the homogenized element.

The continuity conditions at the crack tip cross section on $\varphi_{2}$ and $w_{0}, 2$, Eq. (13), forces $\varphi_{2}+w_{0}, 2$ to be zero at the crack tip and the local rotations to coincide there on both sides. In the intact region away from the crack tip, on the other hand, the local rotations generally differ, since $\Lambda_{22}^{1} \neq 0$ and $\varphi_{2}+w_{0,2} \neq 0$.

Force and moment sub-resultants acting in the first and second layers in the delaminated and intact portions of the homogenized element in Fig. 3.b are defined using the local coordinates as: 


$$
\begin{aligned}
& {\left[{ }^{(i)} N_{22}\left(x_{2}\right),{ }^{(i)} M_{22}^{b}\left(x_{2}\right)\right]=\int_{-}^{(i)} h / 2{ }^{(i)} h / 2 \sigma_{22}\left(x_{2},{ }^{(i)} X_{3}\right)\left[1,{ }^{(i)} X_{3}\right] d^{(i)} X_{3}} \\
& { }^{(i)} Q_{2 g}\left(x_{2}\right)=\int_{-{ }^{(i)} h / 2}^{(i)} h / 2{ }^{(i)} \sigma_{23}^{\text {post }}\left(x_{2},{ }^{(i)} X_{3}\right) d^{(i)} X_{3}
\end{aligned}
$$

for $i=1$ and 2 . They are related to the global resultants acting at the end of the homogenized element, Fig. 3.b, through simple equilibrium, e.g. ${ }^{(1)} N_{22}\left(x_{2}=-a\right)+{ }^{(2)} N_{22}\left(x_{2}=-a\right)=N_{22} \quad$ and ${ }^{(1)} N_{22}\left(x_{2}=c\right)+{ }^{(2)} N_{22}\left(x_{2}=c\right)={ }^{0} N_{22}$, and in the delaminated region coincide with the "actual" forces acting on the "actual" element in Fig. 3.a.

\section{Local axial and bending fields}

In the delaminated portion of the homogenized element in Fig. 3, the dominant term of the shear deformations in the first layer vanishes, $\left[\begin{array}{c}0 \\ \gamma]\end{array}\right]=0$, and the longitudinal displacements in the layers, Eq. (8), can be expressed in terms of the displacement jump using Eq. (6):

$$
\begin{aligned}
& { }^{(1)} v_{2}\left(x_{2}, x_{3}\right)=v_{02}\left(x_{2}\right)+x_{3} \varphi_{2}\left(x_{2}\right) \\
& { }^{(2)} v_{2}\left(x_{2}, x_{3}\right)=\left[v_{02}\left(x_{2}\right)+\hat{v}_{2}\left(x_{2}\right)\right]+x_{3} \varphi_{2}\left(x_{2}\right) \text { for }-a \leq x_{2} \leq 0
\end{aligned}
$$

The equations above and the local coordinates are used to redefine the axial stresses and strains in the delaminated region, Eq. (10). Substituting the axial stresses into the first equation (22), performing the integration and some algebraic manipulations [44], yield axial strains and stresses in the layers $i$ $=1,2$ of the delaminated region in terms of the normal force and moment sub-resultants:

$$
\begin{aligned}
& { }^{(i)} \varepsilon_{22}\left(x_{2},{ }^{(i)} X_{3}\right)=\frac{{ }^{(i)} N_{22}}{{ }^{(i)} \bar{E}_{2}{ }^{(i)} h}+{ }^{(i)} X_{3} \frac{12^{(i)} M_{22}^{b}\left(x_{2}\right)}{{ }^{(i)} \bar{E}_{2}\left({ }^{(i)} h\right)^{3}} \text { for }-a \leq x_{2} \leq 0 \\
& { }^{(i)} \sigma_{22}\left(x_{2},{ }^{(i)} X_{3}\right)=\frac{{ }^{(i)} N_{22}}{{ }^{(i)} h}+{ }^{(i)} X_{3} \frac{12^{(i)} M_{22}^{b}\left(x_{2}\right)}{\left({ }^{(i)} h\right)^{3}} \quad \text { for }-a \leq x_{2} \leq 0 \\
& \varphi_{2}, 2\left(x_{2}\right)=\frac{12^{(i)} M_{22}^{b}\left(x_{2}\right)}{{ }^{(i)} \bar{E}_{2}\left({ }^{(i)} h\right)^{3}} \quad \text { for }-a \leq x_{2} \leq 0
\end{aligned}
$$

The equations have the same form of those of the equivalent single layer theory for homogeneous layers. This result highlights the capability of the homogenized approach to describe the local discontinuous fields in the delaminated region of the plate from just three global kinematic variables. 
In the intact portion of the element in Fig. 3.c, the constitutive equations of the model, Eq. (17), modify since $B_{22}=0$ :

$$
\begin{array}{ll}
{ }^{(0)} N_{22}\left(x_{2}\right)=A_{22} v_{02},{ }_{2}\left(x_{2}\right)+C^{0 S} \gamma_{, 2}\left(x_{2}\right) & \text { for } 0 \leq x_{2} \leq c \\
{ }^{(0)} M_{22}^{b}\left(x_{2}\right)=D_{22} \varphi_{2},{ }_{2}\left(x_{2}\right)+C^{1 S} \gamma_{, 2}\left(x_{2}\right) & \text { for } 0 \leq x_{2} \leq c
\end{array}
$$

The last terms are not present in a homogeneous plate, where $C^{0 S}=C^{1 S}=0$; in a bi-material plate they becomes negligible at a sufficient distance from the crack tip since the exponential terms in the solution of $\gamma$ in Eq. (20) decrease moving away from the crack tip in order for $\gamma$ to become independent of $x_{2}$, as is required by the geometry and loading conditions (shear is constant along the ligament ahead of the crack tip). For sufficiently long $c, \gamma$ is constant with respect to $x_{2}$, which implies that $\gamma_{,_{2}}=0$. As a consequence, the axial strain and stress in the intact region at a sufficient distance from the crack tip are defined in terms of the global normal force and bending moment resultants, Eqs. (10) and (25), by:

$$
\begin{aligned}
& { }^{(k)} \varepsilon_{22}\left(x_{2}=c, x_{3}\right)=\frac{{ }^{(0)} N_{22}}{A_{22}}+x_{3} \frac{{ }^{(0)} M_{22}^{b}}{D_{22}} \\
& { }^{(k)} \sigma_{22}\left(x_{2}=c, x_{3}\right)={ }^{(k)} \bar{E}_{2}\left[\frac{{ }^{(0)} N_{22}}{A_{22}}+x_{3} \frac{{ }^{(0)} M_{22}^{b}}{D_{22}}\right]
\end{aligned}
$$

As for the delaminated layers, at a sufficient distance from the crack tip in the intact region, the constitutive equations have the same form of those of the classical plate theory.

\section{Local shear fields}

The transverse shear strains in the layers, Eq. (10), can be rewritten in terms of the local rotations of the layers in Eq. (21). This yields an expression which is similar to that of the first order shear deformation theory and applies to the delaminated and intact regions of the plate:

$$
2^{(k)} \varepsilon_{23}\left(x_{2}, x_{3}\right)=w_{0}, 2\left(x_{2}\right)+{ }^{(k)} \varphi_{2}\left(x_{2}\right)
$$


The shear strains are constant in the thickness of each layer and vanish in the delaminated portion of the plate where $w_{0,2}+{ }^{(k)} \varphi_{2}=0$. Shear strains, ${ }^{(k)} \varepsilon_{23}^{\text {post }}$, related to the a posteriori calculated shear stresses which satisfy local equilibrium, ${ }^{(k)} \sigma_{23}^{\text {post }}$, have already been defined in Eq. (11).

For the derivation of the energy release rate in Sect. 3.3 in terms of crack tip force and moment sub-resultant, it is convenient to introduce, following the Jourawsky's method, global strain measures energetically associated to the generalized shear resultant, Eq. (14), and sub-resultants, (22), acting at the end sections at the coordinates $x_{2}=-a, c$. This is done by imposing:

$$
\frac{1}{2}\left(2^{(i)} \varepsilon_{23 g}{ }^{(i)} Q_{2 g}\right)=\frac{1}{2} \int_{-{ }^{(i)} h / 2}^{(i)} / 22^{(i)} \varepsilon_{23}^{\text {post }{ }^{(i)}} \sigma_{23}^{\text {post }} d^{(i)} X_{3}
$$

where ${ }^{(i)} \sigma_{23}^{\text {post }}$ are ${ }^{(i)} \varepsilon_{23}^{\text {post }}$ are related through Eq. (11), (24) to the axial and moment sub-resultants, in the delaminated region, and to the axial and moment resultants, Eqs. (11), , (26) at $x_{2}=c$ in the intact region away from the crack tip. Global equilibrium, Eq. (12), and local equilibrium in the homogeneous delaminated layers, ${ }^{(i)} M_{22}^{b},_{2}-{ }^{(i)} Q_{2 g}=0$, then yields [44]:

$$
2^{(i)} \varepsilon_{23 g}=\frac{{ }^{(i)} Q_{2 g}}{{ }^{(i)} k_{44}{ }^{(i)} G_{23}{ }^{(i)} h} \quad \text { for } i=1,2 \text { and } x_{2} \leq 0 ; \quad 2^{(0)} \varepsilon_{23 g}\left(x_{2}=c\right)=\frac{{ }^{(0)} Q_{2 g}\left(x_{2}=c\right)}{{ }^{(0)} k_{44} \sum_{i=1,2}{ }^{(i)} G_{23}{ }^{(i)} h}
$$

with:

$$
\begin{aligned}
& { }^{(i)} k_{44}=5 / 6 \text { for } i=1,2 ; \\
& { }^{(0)} k_{44}=\left[\left(C_{22}^{2}\right)^{-2} \sum_{k=1}^{2}{ }^{(k)} G_{23}{ }^{(k)} h\left(\int_{-d}^{-d+h_{1}} l\left(x_{3}\right)^{2} /{ }^{(1)} G_{23} d x_{3}+\int_{-d+h_{1}}^{-d+h_{1}+h_{2}} m\left(x_{3}\right)^{2} /{ }^{(2)} G_{23} d x_{3}\right)\right]^{-1}
\end{aligned}
$$

and

$$
\begin{aligned}
& l\left(x_{3}\right)=\frac{1}{2}\left(-{ }^{(1)} \bar{E}_{2} x_{3}{ }^{2}+{ }^{(1)} \bar{E}_{2} d^{2}\right) \quad ; \quad d={ }^{(1)} e+{ }^{(1)} h / 2 \\
& m\left(x_{3}\right)=\frac{1}{2}\left[-{ }^{(2)} \bar{E}_{2} x_{3}{ }^{2}+{ }^{(1)} \bar{E}_{2} d^{2}+\left({ }^{(2)} \bar{E}_{2}-{ }^{(1)} \bar{E}_{2}\right)\left({ }^{(1)} h-d\right)^{2}\right]
\end{aligned}
$$

(Fig. 3.c for the notation). The local shear correction factors, ${ }^{(i)} k_{44}$, coincide with those typically defined for classical first order theories. This is expected since the axial stresses, which are used in 
Eq. (28), are related to the normal and moment resultants/sub-resultants through relationships, Eqs. (24)(26), which coincide with those of the classical theories.

\section{Energy release rate in layered beams with single and multiple delaminations under Mode II dominant conditions}

The general solution of the homogenized model, Eq. (19), and the boundary conditions in Eq. (48), are used to define the energy release rate for the collinear propagation of delamination cracks in simply and multiply delaminated layered beams under mode II dominant conditions, Fig. 4. Problems characterized by mixed-mode conditions could be studied similarly using the extended version of the multiscale model presented in [19], which accounts also for the interfacial opening displacements.

The energy release rate is calculated using the compliance method, Sect. 3.1, and through different applications of the J-Integral, Sect. 3.2. For the special case of homogeneous and bi-material beams with a single delamination, closed form solutions are derived in terms of crack tip force and moment sub-resultants, Sect. 3.3

\subsection{Energy release rate: compliance method}

In order to apply the homogenized model to the specimens in Fig. 4, cohesive interfaces governed by the interfacial traction law in Eq. (4) are introduced along the crack planes. The numerical values for the interfacial stiffnesses in the intact and delaminated portions are chosen as $K_{S}^{k(k)} h / \bar{E}_{2}=10^{3}$ and $K_{S}^{k(k)} h / \bar{E}_{2}=10^{3}=10^{-10}$, respectively, Fig. 2; these values are respectively large and small enough values to avoid numerical problems and ensure the convergence of the solution. The specimens are then homogenized, Fig. 4, discretized into portions separated by the coordinates which define the crack tips and concentrated loads and the coefficients which describe the homogenized stiffnesses of the different portions are calculated, Eq. (47)(49). The unknown integration constants of the general solutions in Eq. (19) are calculated by imposing boundary and continuity conditions. The boundary conditions are: $\tilde{w}_{0}=\tilde{M}_{2}^{b}=\tilde{M}_{2}^{z S}=0$ and $\tilde{N}_{2}=0$ or $\tilde{v}_{02}=0$ at the simple supports, Fig. 4.a,b; $\tilde{w}_{0}=\tilde{\varphi}_{2}=\tilde{v}_{02}=0$ at the clamped ends, Fig. 4.c; $\tilde{N}_{2}=\tilde{M}_{2}^{b}=\tilde{M}_{2}^{z S}=0$

and $\tilde{Q}_{2 g} \neq 0$ at the free ends subjected to concentrated transverse loads, Fig. 4.c. Continuity conditions are imposed on the global variables $v_{02}, \varphi_{2}, w_{0}, w_{0},{ }_{2}, N_{22}, M_{22}^{b}, Q_{2 g}$ and $M_{22}^{z S}$.

Using the solution of the homogenized model, the energy release rate is calculated as the variation of the total potential energy for unit collinear crack advancement: 
$G_{I I}=-\frac{d \prod}{d a}=\frac{P^{2}}{2} \frac{d C}{d a}$

with $\Pi$ the total potential energy, $P$ the load applied per unit width and $C=w_{0}\left(x_{2}=L\right) / P$ the load point displacement due to a unit load. Using Eq. (31) allows to verify the capability of the homogenized model to define transverse displacements in cracked beams.

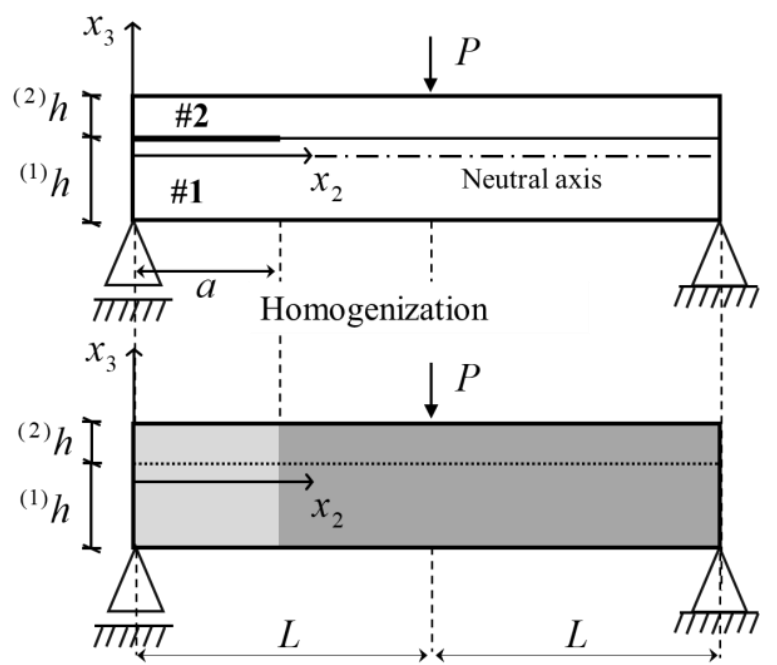

(a)

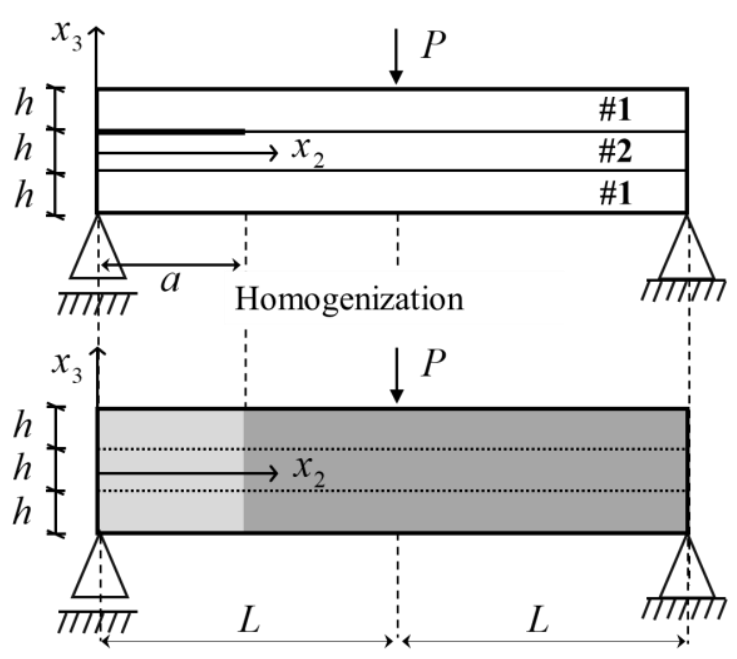

(b)

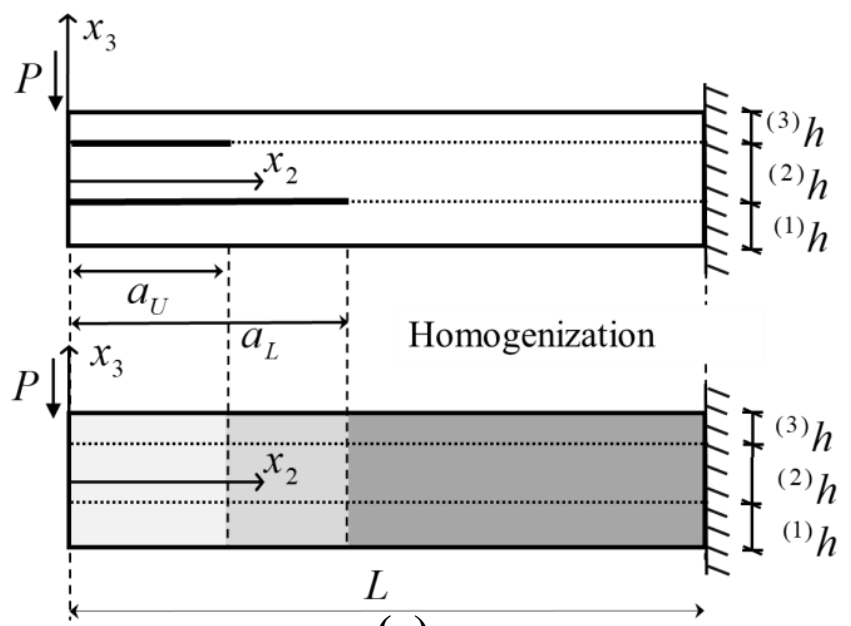

(c)

Fig. 4. (a) Three-point bend-beam and homogenized model, used for the beams in Table 1. (b) Three-point bend-beam with layered (sandwich) structure and homogenized model, Table 4. (c) Cantilever bend-beam with two delaminations and homogenized model, used for the beams in Table 7. 


\subsection{Energy release rate: J-Integral}

In the beams with a single delamination, Figs. 4.a,b, the energy release rate for the collinear propagation of the delamination is also calculated using the J-integral along a path surrounding the crack tip, shown in Fig. 3.b:

$$
G_{I I}=J=\int_{\Gamma}\left(W d x_{3}-\sigma_{i j} n_{j} v_{i}, 2 d \Gamma\right)
$$

for $i, j=2,3$, with $\Gamma$ the contra-clockwise path surrounding the crack tip and $d \Gamma$ an element of arc length along $\Gamma ; W=\left(\sigma_{22} \varepsilon_{22}+2 \sigma_{23} \varepsilon_{23}\right) / 2$ is the strain energy density, $n_{j}$ is the component of the unit outward normal vector and $\sigma_{i j} n_{j}$ are the tractions along the contour. The terms in the J-integral are obtained from the solution of the homogenized model, Eqs. (19)(48). The requirement to obtain an accurate prediction of the energy release rate is that the quantities in the J-integral must be a posteriori calculated values, namely $W=\left(\sigma_{22} \varepsilon_{22}+2 \sigma_{23}^{\text {post }} \varepsilon_{23}^{\text {post }}\right) / 2, \quad \sigma_{i j} n_{j}=\sigma_{i j}^{\text {post }} n_{j} \quad$ components and ${ }^{(i)} v_{3}^{\text {post }}, 2={ }^{(i)} 2 \varepsilon_{23}^{\text {post }}-{ }^{(i)} \varphi_{2}$, after Eq. (27).

\section{J-Integral along a path following the delamination surfaces}

To verify the capability of the homogenized model to accurately predict crack surface displacements, the energy release rate is also calculated using a J-Integral path which follows the delamination surfaces, Fig. 5.a. Using Bückner's superposition principle and the procedure in [45], and since the crack surfaces can only slide with no friction, Eq. (31) applied to Fig. 5.c yields:

$$
J=-\int_{x_{2}=-a}^{x_{2}=0} \tau\left(x_{2}\right)^{(1)} v_{2}, 2\left(x_{2}, x_{3}=x_{3}^{1}\right) d x_{2}-\int_{x_{2}=0}^{x_{2}=-a} \tau\left(x_{2}\right)^{(2)} v_{2}, 2\left(x_{2}, x_{3}=x_{3}^{1}\right) d x_{2}
$$

where $-\tau\left(x_{2}\right)$ are the shear tractions generated at level of the cracked interface in the intact homogenized element in Fig. 5. The Mode II energy release rate is:

$$
G_{I I}=\int_{x_{2}=-a}^{x_{2}=0} \tau\left(x_{2}\right) \hat{v}_{2,2} d x_{2}={ }^{(1)} \sigma_{23}^{\text {post }}\left(x_{3}=x_{3}^{1}\right)\left[\hat{v}_{2}\left(x_{2}=-a\right)-\hat{v}_{2}\left(x_{2}=0\right)\right]
$$

since $\tau\left(x_{2}\right)=-{ }^{(1)} \sigma_{23}^{\text {post }}\left(x_{3}=x_{3}^{1}\right)$ does not depend on $x_{2}$ for $0 \leq x_{2} \leq a$. 
The energy release rate calculated through Eq. (34) relies on accurate calculations of the relative crack displacement. Concepts of perturbation analysis, already anticipated in the previous section for the bi-material case, show that zero order expansion of the relative crack displacement for $K_{s}^{1} \rightarrow 0$, which is the dominant term in the delaminated region, is $\left[\hat{v}_{2}\right]=[\gamma]^{(2)} G_{23}\left(1+\Lambda_{22}\right)$ and depends on the first order expansion of $\gamma=\varphi_{2}+w_{0},,_{2}$. The presence of terms which do not depend on $x_{2}$ in the first order expansion of $\gamma$ [44] implies that the relative crack displacement does not vanish at the crack tip. This results, which is a consequence of the imposition of continuity conditions only on global quantities, has no effect on the energy release rate in Eq. (34), since the terms of $\hat{v}_{2}$ which are independent of $x_{2}$ cancel in $\hat{v}_{2}\left(x_{2}=-a\right)-\hat{v}_{2}\left(x_{2}=0\right)$.

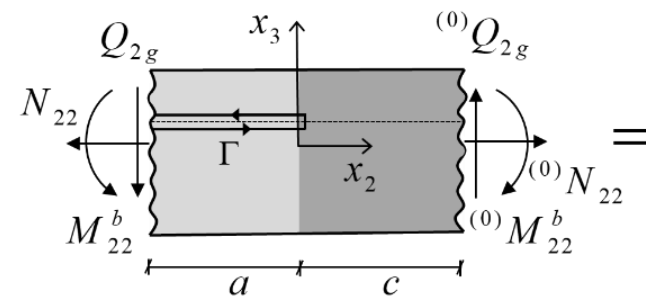

(a)

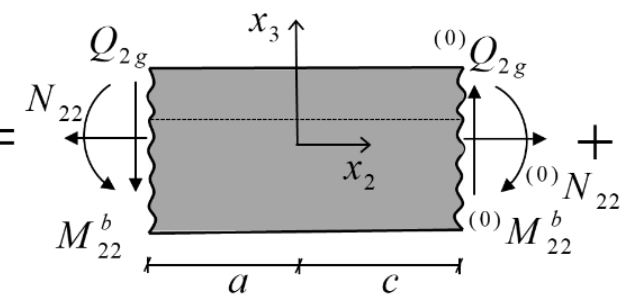

(b)

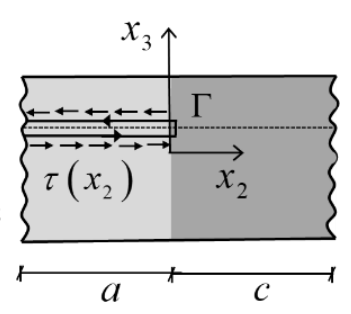

(c)

Fig. 5. Bückner's superposition scheme used to calculate the J-integral along the crack surfaces.

\subsection{Energy release rate: closed form solutions for unidirectional and bi-material beams}

In this section, closed form solutions are derived for the energy release rate of homogeneous and bimaterial beams with a single delamination arbitrarily located in the thickness. The energy release rate is calculated referring to the cracked element in Fig. 3.a subject to force and moment resultants acting at the end sections. The length of crack and ligament, $a$ and $c$, are assumed to be long enough so that the crack tip fields can be described in term of end resultants. The minimum lengths depend on the mismatch of the elastic constants and are on the order of the global thickness of the plate for conventional composites, [46-49]. The assumption allows comparison of the results with accurate 2D solutions from the literature. The derivation uses the local fields discussed in Sect. 2.3 and the JIntegral in Eq. (32).

The components of the J-Integral along the paths $\Gamma_{4}$ and $\Gamma_{5}$ in Fig. 3.b are $J_{4}=J_{5}=0$, since the upper and lower surfaces of the element are traction-free. The components of the J-Integral along the paths $\Gamma_{i}$ for $\mathrm{i}=1,2$, are obtained using Eqs. (32)(10) : 


$$
\begin{aligned}
& J_{i=1,2}\left(x_{2}=-a\right)=\frac{1}{2} \int_{-\frac{{ }^{(i)} h}{2}}^{{ }^{(i)} h} \sigma_{22}{ }^{(i)} \varepsilon_{22} d^{(i)} X_{3}-\frac{1}{2} \int_{-\frac{(i) h}{2}}^{\frac{{ }^{(i)} h}{2}} 2^{(i)} \sigma_{23}{ }^{(i)} \varepsilon_{23} d^{(i)} X_{3}+ \\
& +\int_{-\frac{(i)}{2}}^{\frac{{ }^{(i)} h}{2}}{ }^{(i)} \sigma_{23}{ }^{(i)} v_{3}, 2 d^{(i)} X_{3}
\end{aligned}
$$

The equation is elaborated by noting that ${ }^{(i)} v_{3,2}=w_{0},_{2}=2^{(i)} \varepsilon_{23}-{ }^{(i)} \varphi_{2}$, after Eq. (27), introducing a posteriori calculated shear stresses and strains, ${ }^{(i)} \sigma_{23}={ }^{(i)} \sigma_{23}^{\text {post }}$ and ${ }^{(i)} \varepsilon_{23}={ }^{(i)} \varepsilon_{23}^{\text {post }}$, Eq. (11), and using the relationships in Eqs. (28)(29) and Eq. (24). This yields:

$$
J_{i=1,2}\left(x_{2}=-a\right)=\frac{1}{2}\left(\frac{\left[{ }^{(i)} N_{22}\right]^{2}}{{ }^{(i)} \bar{E}_{2}{ }^{(i)} h}+\frac{12\left[{ }^{(i)} M_{22}^{b}\right]^{2}}{{ }^{(i)} \bar{E}_{2}\left({ }^{(i)} h\right)^{3}}+\frac{\left[{ }^{(i)} Q_{2 g}\right]^{2}}{{ }^{(i)} k_{44}{ }^{(i)} G_{23}{ }^{(i)} h}-2^{(i)} \varphi_{2}{ }^{(i)} Q_{2 g}\right)
$$

Similar considerations are applied along the path $\Gamma_{3}$, which yield (see [44] for details):

$$
J_{3}\left(x_{2}=c\right)=-\frac{1}{2}\left(\frac{\left[{ }^{(0)} N_{22}\right]^{2}}{A_{22}}+\frac{\left[{ }^{(0)} M_{22}^{b}\right]^{2}}{D_{22}}+\frac{\left[{ }^{(0)} Q_{2 g}\right]^{2}}{{ }^{(0)} k_{44} \sum_{i=1,2}{ }^{(i)} G_{23}{ }^{(i)} h}\right)+{ }^{(1)} \varphi_{2}{ }^{(1)} Q_{2 g}+{ }^{(2)} \varphi_{2}{ }^{(2)} Q_{2 g}
$$

Summing up the contribution is Eqs. (36)(37), the energy release rate in the homogenized element in Fig. 3.b is:

$$
\begin{aligned}
& G_{I I}=J=\frac{1}{2}\left[\left.\sum_{i=1}^{2}\left(\frac{\left[{ }^{(i)} N_{22}\right]^{2}}{{ }^{(i)} \bar{E}_{2}{ }^{(i)} h}+\frac{12\left[{ }^{(i)} M_{22}^{b}\right]^{2}}{{ }^{(i)} \bar{E}_{2}\left({ }^{(i)} h\right)^{3}}+\frac{\left[{ }^{(i)} Q_{2 g}\right]^{2}}{{ }^{(i)} k_{44}{ }^{(i)} G_{23}{ }^{(i)} h}-2^{(i)} \varphi_{2}{ }^{(i)} Q_{2 g}\right)\right|_{x_{2}=-a}\right. \\
& \left.\left.\left(\frac{\left[{ }^{(0)} N_{22}\right]^{2}}{A_{22}}+\frac{\left[{ }^{(0)} M_{22}^{b}\right]^{2}}{D_{22}}+\frac{\left[{ }^{(0)} Q_{2 g}\right]^{2}}{{ }^{(0)} k_{44} \sum_{i=1,2}{ }^{(i)} G_{23}{ }^{(i)} h}-2^{(1)} \varphi_{2}{ }^{(1)} Q_{2 g}-2^{(2)} \varphi_{2}{ }^{(2)} Q_{2 g}\right)\right|_{X_{2}=c}\right]
\end{aligned}
$$

where ${ }^{(i)} N_{22},{ }^{(i)} M_{22}^{b}$ and ${ }^{(i)} Q_{2 g}$ for $i=0,1$ and 2 , are the force and moment sub-resultants acting on the different arms, Eq. (22), and ${ }^{(1)} \varphi_{2}=\varphi_{2}$ and ${ }^{(2)} \varphi_{2}$ are the local rotations of the first and second layers at the edges of the homogenized element, given in Eq. (21). 
When the first and second layers of the element in Fig. 3.a have the same elastic constants, the neutral axis of the intact section is at mid-thickness and the following relationships hold: $\Lambda_{22}^{1}=0$ , ${ }^{(1)} \varphi_{2}={ }^{(2)} \varphi_{2}=\varphi_{2},{ }^{(1)} e={ }^{(2)} h / 2,{ }^{(2)} e={ }^{(1)} h / 2$ and ${ }^{(i)} k_{44}=k_{44}=5 / 6$ for $i=0,1,2$. The energy release rate in Eq. (38) then coincides with that derived in [21][48] using structural mechanics considerations.

\section{Particularization to crack tip quantities: homogeneous element}

For a homogeneous beam, Eq. (38) can be redefined in terms of crack tip quantities by noting that the normal and shear resultants and sub-resultants at the crack tip coincide with those at the element ends, Fig. 3.b, while the bending moments are ${ }^{(i)} M_{22}^{b}\left(x_{2}=0\right)={ }^{(i)} M_{22}^{b}\left(x_{2}=-a\right)+{ }^{(i)} Q_{2 g} a$ for $\mathrm{i}=1,2$ and ${ }^{(0)} M_{22}^{b}\left(x_{2}=0\right)={ }^{(0)} M_{22}^{b}\left(x_{2}=c\right)-{ }^{(0)} Q_{2 g} c$. In addition, the rotations of the end sections of the layer are calculated by integrating the third equation in (24) from $x_{2}=-a$ to $x_{2}=0$, and the second equation in (25) from $x_{2}=0$ to $x_{2}=c$ :

$$
\begin{aligned}
& { }^{(i)} \varphi_{2}\left(x_{2}=-a\right)={ }^{(i)} \varphi_{2}\left(x_{2}=0^{-}\right)-\left[\frac{12^{(i)} M_{22}^{b}\left(x_{2}=-a\right) a}{\bar{E}_{2}\left({ }^{(i)} h\right)^{3}}+\frac{6^{(i)} Q_{2 g}\left(x_{2}=-a\right) a^{2}}{\bar{E}_{2}\left({ }^{(i)} h\right)^{3}}\right] \\
& \varphi_{2}\left(x_{2}=c\right)=\varphi_{2}\left(x_{2}=0^{+}\right)+\frac{12^{(0)} M_{22}^{b}\left(x_{2}=c\right) c}{\bar{E}_{2}\left({ }^{(1)} h+{ }^{(2)} h\right)^{3}}-\frac{6^{(0)} Q_{2 g}\left(x_{2}=c\right) c^{2}}{\bar{E}_{2}\left({ }^{(1)} h+{ }^{(2)} h\right)^{3}}
\end{aligned}
$$

The energy release rate in terms of crack tip quantities becomes:

$$
\begin{aligned}
& G_{I I}=\frac{1}{2}\left\{\left.\sum_{i=1}^{2}\left[\left(\frac{\left[{ }^{(i)} N_{22}\right]^{2}}{\bar{E}_{2}{ }^{(i)} h}+\frac{12\left[{ }^{(i)} M_{22}^{b}\right]^{2}}{\bar{E}_{2}\left({ }^{(i)} h\right)^{3}}+\frac{\left[{ }^{(i)} Q_{2 g}\right]^{2}}{k_{44} G_{23}{ }^{(i)} h}\right)+2^{(i)} Q_{2 g} \Delta \varphi_{i}\right]\right|_{x_{2}=0^{-}}\right. \\
& \left.-\left.\left(\frac{\left[{ }^{(0)} N_{22}\right]^{2}}{\bar{E}_{2}\left({ }^{(1)} h+{ }^{(2)} h\right)}+\frac{12\left[{ }^{(0)} M_{22}^{b}\right]^{2}}{\bar{E}_{2}\left({ }^{(1)} h+{ }^{(2)} h\right)^{3}}+\frac{\left[{ }^{(0)} Q_{2 g}\right]^{2}}{k_{44} G_{23}\left({ }^{(1)} h+{ }^{(2)} h\right)}\right)\right|_{x_{2}=0^{+}}\right\}
\end{aligned}
$$

where $\Delta \varphi_{i}=\varphi_{2}\left(x_{2}=0^{+}\right)-{ }^{(i)} \varphi_{2}\left(x_{2}=0^{-}\right)$for $i=1,2$ define the relative rotations of the arms at the crack tip, also known in the literature as crack tip root-rotations [46,49][51]. Imposition of the continuity condition on the global rotation at the crack tip cross section of the homogenized model implies ${ }^{(0)} \varphi_{2}={ }^{(1)} \varphi_{2}={ }^{(2)} \varphi_{2}=\varphi_{2}$ and $\Delta \varphi_{i}=0$ at $x_{2}=0$, which yields: 


$$
\begin{aligned}
& G_{I I}=\frac{1}{2}\left[\left.\sum_{i=1}^{2}\left(\frac{\left[{ }^{(i)} N_{22}\right]^{2}}{\bar{E}_{2}{ }^{(i)} h}+\frac{12\left[{ }^{(i)} M_{22}^{b}\right]^{2}}{\bar{E}_{2}\left({ }^{(i)} h\right)^{3}}+\frac{\left[{ }^{(i)} Q_{2 g}\right]^{2}}{k_{44} G_{23}{ }^{(i)} h}\right)\right|_{x_{2}=0^{-}}\right. \\
& -\left.\left(\frac{\left[{ }^{(0)} N_{22}\right]^{2}}{\bar{E}_{2}\left({ }^{(1)} h+{ }^{(2)} h\right)}+\frac{12\left[{ }^{(0)} M_{22}^{b}\right]^{2}}{\bar{E}_{2}\left({ }^{(1)} h+{ }^{(2)} h\right)^{3}}+\frac{\left[{ }^{(0)} Q_{2 g}\right]^{2}}{k_{44} G_{23}\left({ }^{(1)} h+{ }^{(2)} h\right)}\right)\right|_{x_{2}=0^{+}}
\end{aligned}
$$

where $k_{44}=5 / 6$. Equation (40) coincides with the accurate 2D solution derived in [49] where the crack tip root rotations $\Delta \varphi_{i}$ are defined as linear functions of the crack tip force and moment resultants through compliance coefficients which depend on the geometry and material properties. The crack tip root rotations describe the near tip deformations and their effect on the energy release rate of the plate can be important in the presence of crack tip shear, also when the crack is long. The homogenized model formulated here, Eq. (41), does not account directly for these effects, which however could be introduced a posteriori as explained in [49]. Additional discussion on this problem can be found in Sect. 4.

\section{Particularization to crack tip quantities: bi-material element}

In the bi-material element, the energy release rate in Eq. (38) can be defined in term of crack tip quantities following a similar procedure. The end rotations of the two delaminated arms, ${ }^{(i)} \varphi_{2}\left(x_{2}=-a\right)$ for $\mathrm{i}=1,2$, coincide with those derived in Eq. (39). The exact definition of the local end rotations of the two layers in the intact part would imply using Eqs. (19)(21). Here, since $c$ is assumed to be sufficiently long, the local end rotations in the intact part are calculated by integrating the curvature $\varphi_{2}, 2$ in the second equation in (25) from $x_{2}=0$ to $x_{2}=c$ and then using ${ }^{(1)} \varphi_{2}=\varphi_{2}$ and ${ }^{(2)} \varphi_{2}=\varphi_{2}+\gamma \Lambda_{22}$, after Eq. (21). This procedure neglects the contribution of the exponential terms in $\gamma$ of Eq. (19), and yields:

$$
\begin{aligned}
& { }^{(1)} \varphi_{2}\left(x_{2}=c\right)=\varphi_{2}\left(x_{2}=0^{+}\right)+\frac{{ }^{(0)} M_{22}^{b}\left(x_{2}=c\right) c}{D_{22}}-\frac{{ }^{(0)} Q_{2 g} c^{2}}{2 D_{22}} \\
& { }^{(2)} \varphi_{2}\left(x_{2}=c\right)=\varphi_{2}\left(x_{2}=0^{+}\right)+\frac{{ }^{(0)} M_{22}^{b}\left(x_{2}=c\right) c}{D_{22}}-\frac{{ }^{(0)} Q_{2 g} c^{2}}{2 D_{22}}+\Lambda_{22} \frac{{ }^{(0)} Q_{2 g}}{k_{44} C_{44}^{P}}\left(1+\frac{C_{22}^{1 S}}{D_{22}}\right)
\end{aligned}
$$

where $k_{44}=5 / 6$ is the global shear correction factor introduced in the equilibrium equations, Eq. (18). The effects of the exponential terms $\gamma$ would affects the local rotation ${ }^{(2)} \varphi_{2}\left(x_{2}=c\right)$ only for 
small values of $c$. The equation above shows that in the intact part of the plate the local rotations of the two layers differ, due to the zigzag enrichment in the third term of ${ }^{(2)} \varphi_{2}$ which comes from the contribution $\gamma \Lambda_{22}$ in Eq. (21) and is therefore related to shear. Noting that ${ }^{(1)} Q_{2 g}={ }^{(0)} Q_{2 g}-{ }^{(2)} Q_{2 g}$, substituting Eq. (42) into Eq. (38) where $\varphi_{2}\left(x_{2}=0^{+}\right)-{ }^{(i)} \varphi\left(x_{2}=0^{-}\right)=0$ for $i=1,2$ due to the continuity conditions, and with some manipulation, yield:

$$
\begin{aligned}
& G_{I I}=\frac{1}{2}\left[\left.\sum_{i=1}^{2}\left(\frac{\left[{ }^{(i)} N_{22}\right]^{2}}{\bar{E}_{2}{ }^{(i)} h}+\frac{12\left[{ }^{(i)} M_{22}^{b}\right]^{2}}{\bar{E}_{2}\left({ }^{(i)} h\right)^{3}}+\frac{\left[{ }^{(i)} Q_{2 g}\right]^{2}}{{ }^{(i)} k_{44} G_{23}{ }^{(i)} h}\right)\right|_{X_{2}=0^{-}}\right. \\
& \left.-\left.\left(\frac{\left[{ }^{(0)} N_{22}\right]^{2}}{A_{22}}+\frac{\left[{ }^{(0)} M_{22}^{b}\right]^{2}}{D_{22}}+\frac{\left[{ }^{(0)} Q_{2 g}\right]^{2}}{{ }^{(0)} k_{44} \sum_{i=1,2}{ }^{(i)} G_{23}{ }^{(i)} h}-2^{(2)} Q_{2 g}\left(x_{2}=c\right) \Lambda_{22} \frac{{ }^{(0)} Q_{2 g}}{k_{44} C_{44}^{P}}\left(1+\frac{C_{22}^{1 S}}{D_{22}}\right)\right)\right|_{x_{2}=0^{+}}\right]
\end{aligned}
$$

The last term on the right hand side describes the work done by the shear sub-resultant in the upper layer, ${ }^{(2)} Q_{2 g}$, on the difference between the local rotation of the upper and lower layers, ${ }^{(2)} \varphi_{2}-{ }^{(1)} \varphi_{2}$, at $x_{2}=c$. All terms in the equation above are to be calculated at the crack tip but for ${ }^{(2)} Q_{2 g}$, which, if calculated from the a posteriori shear stresses may suffer at the crack tip from the presence of a boundary layer. It is therefore preferable to use the value calculated at a sufficient distance from the crack tip. Comparing Eqs. (43) and (41) highlights the effect of the local zigzag enrichment in bimaterial systems. The applications in Sect. 4 will further explain the role of this term.

\section{Applications}

In this section the homogenized model is applied to define energy release rate and analyze crack propagation in the beams in Fig. 4. Homogeneous, bi-material, sandwich and laminated beams with single and multiple delaminations are analyzed. The results are compared with 2D elasticity solutions, with solutions obtained using classical discrete-layer models and with experimental results.

\subsection{Homogeneous and bi-material beams with a single delamination, Fig. 4.a}

The beam with a single delamination in Fig. 4.a is loaded in three-point bending. Three material/geometrical configurations are examined and detailed in Table 1. Beam 1 is homogeneous, orthotropic, with a mid-thickness delamination and represents an End-Notched Flexural (ENF) 
specimen used for Mode II fracture testing of unidirectional composites (see [52] for a description). Beam 2 is homogeneous with unequal thickness layers. Beam 3 is a bi-material beam with equal thickness layers. The delaminations in Beams 2 and 3 are in Mode II dominant conditions and the phase angle, $\psi=\tan ^{-1}\left(\sqrt{G_{I I}} / \sqrt{G_{I}}\right)$, which define a measure of the amount of Mode II to Mode I loading, is defined using the 2D elasticity solutions in [46][47][49] and presented in Table 2.

Using the notation in Fig. 3.b, the homogenized forces in the homogenized beam in Fig. 4.a are calculated as follows. The global force and moment resultants are obtained through simple equilibrium considerations. For $0 \leq x_{2} \leq L$, they are $N_{22}\left(x_{2}\right)={ }^{(0)} N_{22}\left(x_{2}\right)=0$, $M_{22}^{b}\left(x_{2}\right)={ }^{(0)} M_{22}^{b}\left(x_{2}\right)=-P x_{2} / 2$ and $Q_{2 g}\left(x_{2}\right)={ }^{(0)} Q_{2 g}\left(x_{2}\right)=-P / 2 \quad$ The sub-resultants in the delaminated portion of the specimen are obtained by noting that ${ }^{(1)} \varphi_{2}, 2\left(x_{2}\right)={ }^{(2)} \varphi_{2}, 2\left(x_{2}\right)=\varphi_{2}, 2\left(x_{2}\right)$, Eq. (21). This yields:

$$
\frac{{ }^{(2)} M_{22}^{b}\left(x_{2}\right)}{{ }^{(1)} M_{22}^{b}\left(x_{2}\right)}=\frac{{ }^{(2)} Q_{2 g}\left(x_{2}\right)}{{ }^{(1)} Q_{2 g}\left(x_{2}\right)}=\frac{{ }^{(2)} \bar{E}_{2}}{{ }^{(1)} \bar{E}_{2}}\left(\frac{{ }^{(2)} h}{{ }^{(1)} h}\right)^{3}
$$

Since ${ }^{(0)} M_{22}^{b}={ }^{(1)} M_{22}^{b}+{ }^{(2)} M_{22}^{b}$ and ${ }^{(0)} Q_{2 g}={ }^{(1)} Q_{2 g}+{ }^{(2)} Q_{2 g}$, for equilibrium, force and moment subresultants for $0 \leq x_{2} \leq a$ are:

$$
\begin{aligned}
& { }^{(1)} Q_{2 g}\left(x_{2}\right)=-\frac{1}{1+\frac{{ }^{(2)} \bar{E}_{2}}{{ }^{(1)} \bar{E}_{2}}\left(\frac{{ }^{(2)} h}{{ }^{(1)} h}\right)^{3}} \frac{P}{2} ; \quad{ }^{(2)} Q_{2 g}\left(x_{2}\right)=-\frac{\frac{{ }^{(2)} \bar{E}_{2}}{{ }^{(1)} \bar{E}_{2}}\left(\frac{{ }^{(2)} h}{{ }^{(1)} h}\right)^{3}}{1+\frac{{ }^{(2)} \bar{E}_{2}}{{ }^{(1)} \bar{E}_{2}}\left(\frac{{ }^{(2)} h}{{ }^{(1)} h}\right)^{3}} \frac{P}{2} \\
& { }^{(1)} M_{22}^{b}\left(x_{2}\right)={ }^{(1)} Q_{2 g} x_{2} ; \quad{ }^{(2)} M_{22}^{b}\left(x_{2}\right)={ }^{(2)} Q_{2 g} x_{2} \\
& { }^{(1)} N_{22}\left(x_{2}\right)={ }^{(2)} N_{22}\left(x_{2}\right)=0
\end{aligned}
$$

They coincide with those obtained using classical discrete approaches by matching the displacements of the two arms at the support [47].

The energy release rate is calculated in closed form and shown in Table 2, using the material/geometrical properties in Table 1, and Eq. (41), with $k_{44}=5 / 6$, for Beam 1 and 2 and Eq. (43), with $k_{44}={ }^{(1)} k_{44}={ }^{(2)} k_{44}=5 / 6=0.833$ and ${ }^{(0)} k_{44}=0.815$, after Eq. (30), for Beam 3. Table 3 shows relative percent errors between the predictions of the homogenized model and the 2D solutions on varying the normalized crack length, $a / h$. The table also shows relative errors obtained using the other approaches presented in Sect. 3. 
Table 1. Geometrical and material properties of homogeneous and bi-material beams in Fig. 4.a

\begin{tabular}{|l|c|c|c|c|c|c|c|}
\hline Beam & Thickness & $\begin{array}{c}\text { Thickness } \\
\text { ratio }\end{array}$ & \multicolumn{2}{|c|}{ Orthotropic layers } & \multicolumn{2}{c|}{ Isotropic layers } \\
\hline & $H=2 h$ & ${ }^{(2)} h /{ }^{(1)} h$ & $E_{1} / E_{2}=E_{3} / E_{2}$ & $G_{23} / E_{2}$ & $v_{23}, v_{21}, v_{13}$ & ${ }^{(2)} E /{ }^{(1)} E$ & ${ }^{(1)} v,{ }^{(2)} v$ \\
\hline Beam 1 & ${ }^{(1)} h+{ }^{(2)} h$ & 1 & 0.071 & 0.033 & $0.32,0.32,0.45$ & \\
\hline Beam 2 & ${ }^{(1)} h+{ }^{(2)} h$ & 0.5 & 0.071 & 0.033 & $0.32,0.32,0.45$ & & \\
\hline Beam 3 & ${ }^{(1)} h+{ }^{(2)} h$ & 1 & & & & $2 / 3$ & 0.5 \\
\hline
\end{tabular}

Table 2 - Closed forms for the Energy Release Rate in the homogeneous and bi-material beams in Table 1, Fig. 4a.

\begin{tabular}{|c|c|c|c|}
\hline$\frac{G \bar{E}_{2} h}{P^{2}}$ & $\begin{array}{l}\text { Homogenized model } \\
\text { Energy release rate }\end{array}$ & $\begin{array}{c}\text { 2D Elasticity } \\
\text { (Energy Release Rate and Mode Mixity angle) }\end{array}$ & Source \\
\hline Beam 1 & $\frac{9}{16}\left(\frac{a}{h}\right)^{2}$, Eq. (41) & $\begin{array}{l}\frac{9}{16}\left(\frac{a}{h}\right)^{2}\left[1+0.728\left(\frac{h}{a}\right)-0.529\left(\frac{h}{a}\right)^{2}\right] \\
\psi=\tan ^{-1}\left(\sqrt{G_{I I} / G_{I}}\right)==90^{\circ}, \text { Mode II }\end{array}$ & {$[47,49]$} \\
\hline Beam 2 & $\frac{3}{8}\left(\frac{a}{h}\right)^{2}\left[1+1.357\left(\frac{h}{a}\right)^{2}\right]$, Eq. (41) & $\begin{array}{l}\frac{3}{8}\left(\frac{a}{h}\right)^{2}\left[1+1.357\left(\frac{h}{a}\right)^{2}+2.141\left(\frac{h}{a}\right)+0.101\left(\frac{h}{a}\right)^{2}\right] \\
\psi=\tan ^{-1}\left(\sqrt{G_{I I} / G_{I}}\right)=65.2^{\circ}\end{array}$ & [49] \\
\hline Beam 3 & $\begin{array}{l}0.445\left(\frac{a}{h}\right)^{2}\left[1-0.012\left(\frac{h}{a}\right)^{2}+0.21\left(\frac{h}{a}\right)^{2}\right], \\
\text { Eq. (43) }\end{array}$ & $\begin{array}{l}0.445\left(\frac{a}{h}\right)^{2}\left[1-0.012\left(\frac{h}{a}\right)^{2}+0.445\left(\frac{h}{a}\right)+0.073\left(\frac{h}{a}\right)^{2}\right. \\
\psi=\tan ^{-1}\left(\sqrt{G_{I I} / G_{I}}\right)=82.4^{\circ}\end{array}$ & {$[46,49]$} \\
\hline
\end{tabular}

Table 3: Relative error on the energy release rate. Error is between results of homogenized model and 2D solutions.

\begin{tabular}{|c|c|c|c|c|c|c|}
\hline $\begin{array}{l}\text { Relative error against } 2 \mathrm{D} \text { solutions in } \\
\text { Table } 2\end{array}$ & & $\frac{a}{h}=10$ & $\frac{a}{h}=15$ & $\frac{a}{h}=20$ & $\frac{a}{h}=30$ & $\frac{a}{h}=40$ \\
\hline J-integral, Table 2, closed form, Eq. (41) & Beam 1 & $-6.3 \%$ & $-4.4 \%$ & $-3.4 \%$ & $-2.3 \%$ & $-1.8 \%$ \\
\hline $\begin{array}{l}\text { J-Integral (crack surface displacement), } \\
\text { numerical, Eq. (34) }\end{array}$ & Beam 1 & $-6.3 \%$ & $-4.4 \%$ & $-3.4 \%$ & $-2.3 \%$ & $-1.8 \%$ \\
\hline Compliance method, numerical, Eq. (31) & Beam 1 & $-10.2 \%$ & $-6.2 \%$ & $-4.4 \%$ & $-2.8 \%$ & $-2.0 \%$ \\
\hline J-integral, Table 2, closed form, Eq. (41) & Beam 2 & $-17.5 \%$ & $-12.5 \%$ & $-9.7 \%$ & $-6.7 \%$ & $-5.1 \%$ \\
\hline $\begin{array}{l}\text { J-Integral (crack surface displacement), } \\
\text { numerical, Eq. (34) }\end{array}$ & Beam 2 & $-18.6 \%$ & $-13.0 \%$ & $-10.0 \%$ & $-6.8 \%$ & $-5.2 \%$ \\
\hline Compliance method, numerical, Eq. (31) & Beam 2 & $-23.7 \%$ & $-15.4 \%$ & $-11.4 \%$ & $-7.4 \%$ & $-5.5 \%$ \\
\hline J-integral, Table 2, closed form, Eq. (43) & Beam 3 & $-4.1 \%$ & $-2.8 \%$ & $-2.1 \%$ & $-1.4 \%$ & $-1.1 \%$ \\
\hline $\begin{array}{l}\text { J-Integral (crack surface displacement), } \\
\text { numerical, Eq. (34) }\end{array}$ & Beam 3 & $-4.3 \%$ & $-2.9 \%$ & $-2.2 \%$ & $-1.5 \%$ & $-1.1 \%$ \\
\hline Compliance method, numerical, Eq. (31) & Beam 3 & $-4.8 \%$ & $-3.1 \%$ & $-2.2 \%$ & $-1.5 \%$ & $-1.1 \%$ \\
\hline
\end{tabular}




\section{Discussion on the Energy Release Rate}

Beam 1 in Table 1, Fig. 4.a, is homogeneous, orthotropic and anti-symmetric. The delamination is under pure mode II conditions. In this beam, the terms in Eq. (41) which depend on the shear forces, give a zero contribution, due to the symmetry of the geometry about the delamination line. The second and third terms in the square brackets of the 2D solution (Table 2) account for the effects of shear on the near tip deformations generated by the bending moments and the shear forces, respectively. The near tip deformations are described in the 2D solution as relative rotations of the crack tip cross sections on the delaminated and intact side, or root-rotations (see discussion in [49]) Predictions using the closed form solution of the homogeneous model in Table 2 are quite accurate for sufficiently long cracks. The energy release rate in terms of crack surface displacements coincides in this problem with that obtained using the crack tip sub-resultants. Predictions obtained using the compliance method are less accurate, but still acceptable for sufficiently long cracks, due to an underestimation of the specimen compliance as a consequence of neglecting the shear deformations in the delaminated portion.

Beam 2 in Table 1, Fig. 4.a, is homogeneous and asymmetric. Since ${ }^{(1)} h>{ }^{(2)} h$, the delamination surfaces are under compression and the result in Table 2 assumes frictionless contact. The second term of the solution of the homogenized model in Table 2 is due to the effect of the crack tip shear forces on the shear deformations. In the $2 \mathrm{D}$ solution, the third and fourth terms in the square brackets account for the effects of shear on the crack tip root-rotations generated by the bending moments and the shear forces, respectively. The mode mixity phase angle is $\psi=65.2^{\circ}[49]$ and differs substantially from the value corresponding to pure mode II, $90^{\circ}$, since the $2 \mathrm{D}$ elasticity solution implies interpenetration of the crack faces at the crack tip. The relative percent error between the predictions of the homogenized model and the 2D solution is larger than that of the anti-symmetric problem. This is due to the important mode I component and to the simplified description of contact (constrained-contact).

Beam 3 Table 1, Fig. 4.a, is a bi-material beam made of two incompressible isotropic layers. The Dundurs' parameters of the interface are $\alpha=-0.2, \beta=0$. For this geometry and material properties, the delamination surfaces are in compression and the homogenized solution in Table 2 is applicable assuming frictionless contact. The first term on the right hand side of the energy release rate calculated using the homogenized model in Table 2 is due to the crack tip bending moment and the second and third terms describe the effects of the shear resultant on the shear deformations in the layers and of the shear sub-resultant in the upper layer of the intact ligament on the difference between the rotations of the upper and lower layers. The $2 \mathrm{D}$ elasticity reference solution is obtained through an elaboration of the results in [47], in order to separate the different contributions following the 
methodology suggested in [49]. The third and fourth terms in the square brackets account for the effects of shear on the crack tip root-rotations generated by the bending moments and the shear forces, respectively. The mode mixity phase angle is equal to $82.4^{\circ}$ and is very close to $90^{\circ}$, which define mode II conditions. This explain the very good predictions of the model also for short cracks. The solution obtained using the J-Integral along the fracture surface coincide with predictions that fully neglect the effects of shear.

\section{Macro-structural Response - Critical load vs load deflection curve of Beam 1, Table 1, Fig. 4.a}

The macrostructural response of Beam 1 in Table 1, Fig. 4.a is analyzed through the critical load versus load deflection curve shown in Fig. 6, for $L=100 h / 3$ and an initial crack of length $a_{0}=20 h$. The crack propagates when the energy release rate of the homogenized model in Table 2 equals the critical value, $G_{I I C}$, and the critical load for crack propagation is:

$$
\frac{P_{c r}}{\sqrt{G_{I I C} h \bar{E}_{2}}}=\frac{4}{3}\left(\frac{h}{a}\right)
$$

with $a=a_{0}=20 h$ for the initial propagation. The load point displacement is obtained from the solution of the model as $w_{0}\left(x_{2}=L\right)$, Eq. (19). To follow crack propagation, the crack length is then progressively increased and the corresponding critical load calculated using Eq. (46). This cracklength control allows to follow the virtual branch associated to the snap-back instability. The results of the homogenized model are compared with those obtained through a 2D solution of the problem using the 2D energy release rate in Table 2. The results obtained using a classical discrete layer model [8] are also presented in the figure. In this approach, the layers in the delaminated region are modeled separately by the first order shear deformation theory and are allowed to freely slide along each other; the intact portion of the specimen is modeled by the first order shear deformation theory.

The sudden change in the shape of the post-peak curve indicates that the crack has approached the mid-span. There the concentrated load generates a compression region which typically arrests the cracks in quasi static laboratory experiments. Here the solution is presented also for $a>L$, which now defines a stable propagation approaching the limiting solution (dash-dot line) of two separated layers free to slide along each other (see also [7]). 


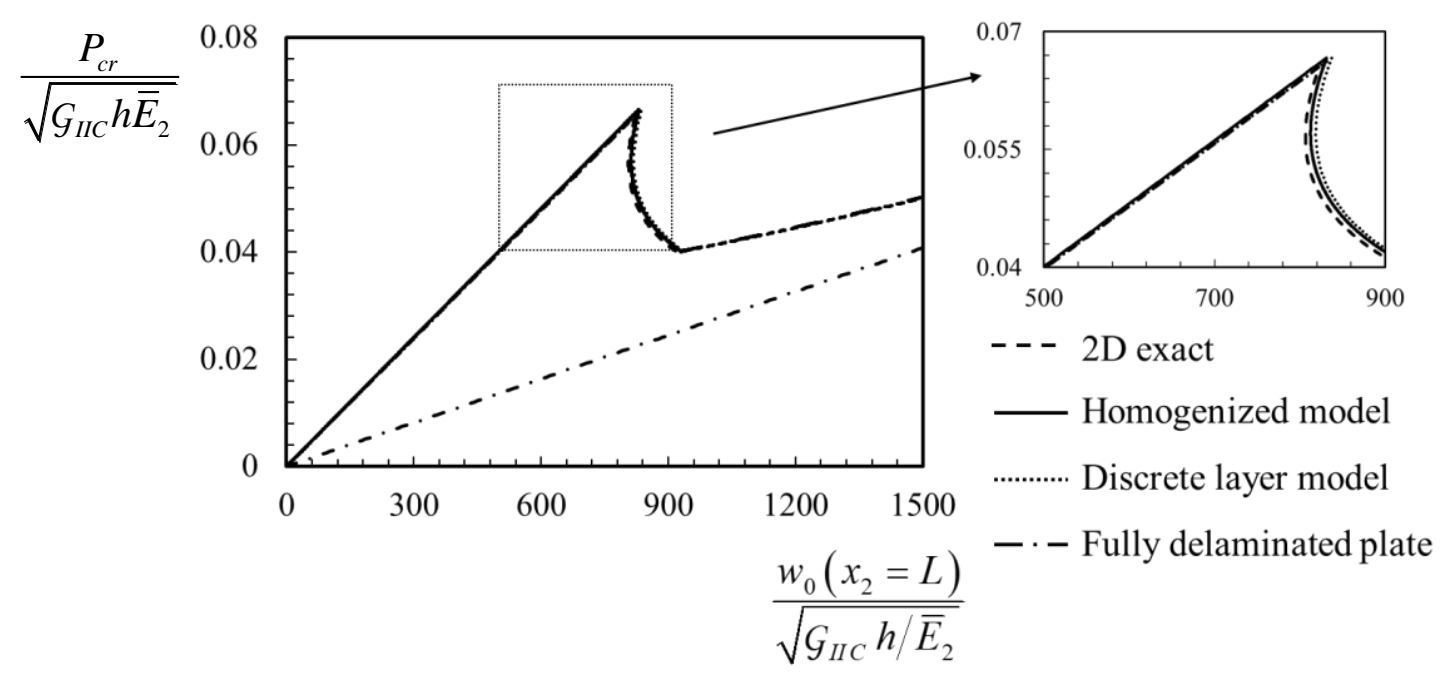

(a)

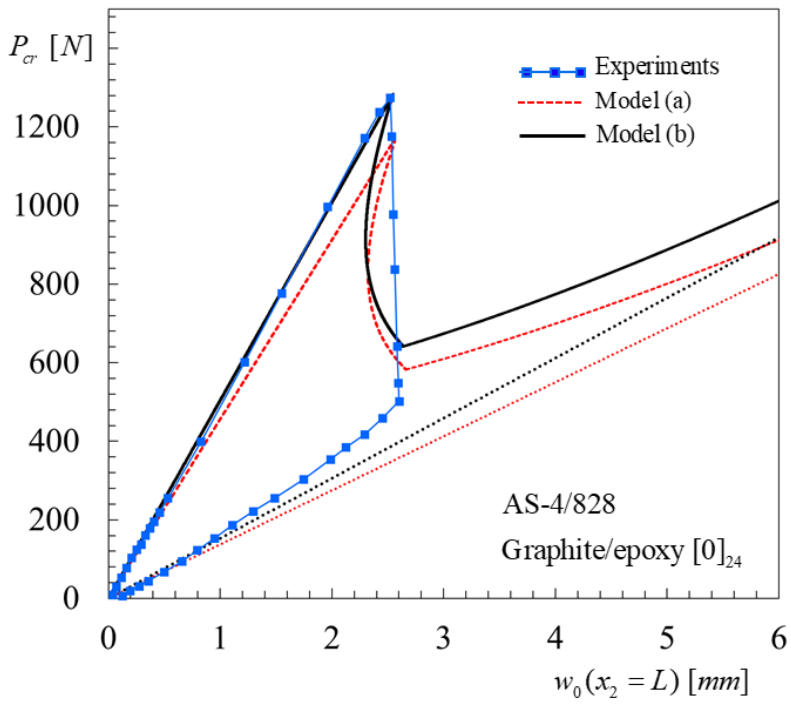

(b)

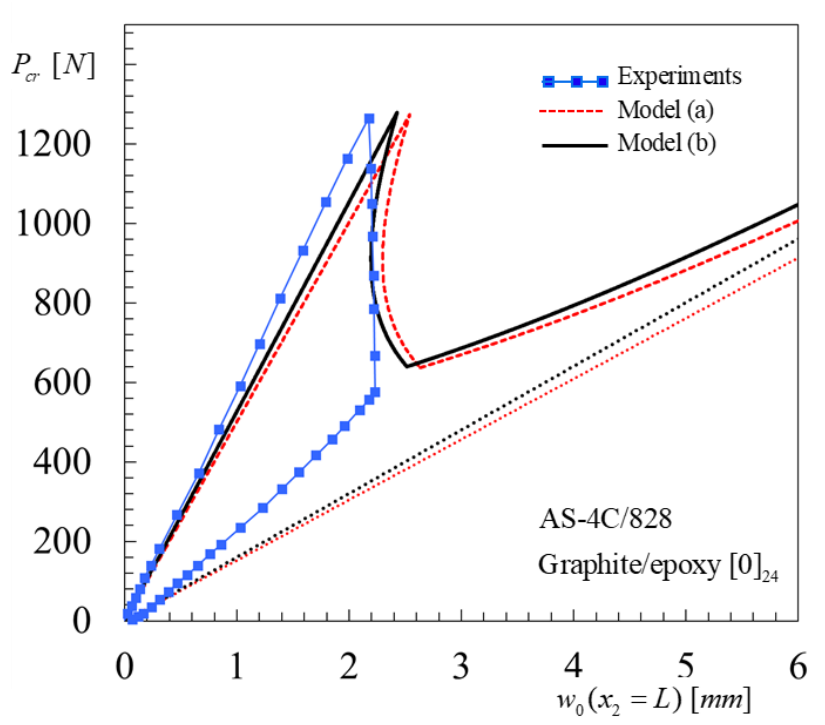

(c)

Fig. 6. (a) Dimensionless diagram of the critical load for crack propagation as function of the load point displacement in: (a) unidirectional ENF specimen in Fig. 4.a, Beam 1, Table 1; (b), (c) unidirectional ENF fracture specimens tested in [53] under displacement control. Model (a) and (b) described in the main text.

\section{Macro-structural Response - Comparison with experimental results}

The diagrams in Figs. 6.b,c show the critical load for crack propagation in two ENF specimens made of Graphite/epoxy $[0]_{24}$ laminates tested in $[53,54]$. The geometry is defined by : $\mathrm{L}=50 \mathrm{~mm}, 2 \mathrm{~h}=$ $3.4 \mathrm{~mm}, a_{0}=25 \mathrm{~mm}, \mathrm{~b}=25 \mathrm{~mm}$ (width), Fig. 4.a. The material of the beam in Fig. 6.b is a graphite/epoxy AS-4/828 with $E_{2}=139 \pm 16.7 \mathrm{GPa}$ and $G_{23}=6 \mathrm{GPa}$, defined from flexural tests on the laminate [54], and Mode II fracture energy $G_{I I C}=1.04 \pm 0.17 \mathrm{~N} / \mathrm{mm}$, calculated in [53] using the 
compliance method. The material of the beam in Fig. 6.c is a graphite/epoxy AS-4C/828 with $E_{2}=158 \pm 5.06 \mathrm{GPa}$ and $G_{23}=6 \mathrm{GPa}$ and $G_{I I C}=1.15 \pm 0.13 \mathrm{~N} / \mathrm{mm}$.

Two theoretical curves are shown in each diagrams. The curves in Fig. 6.b have been obtained using the average values of the elastic constants and energy release rate, red dashed lines Model (a), and the maximum values, black solid lines Model (b). In Fig. 6.c the red curve corresponds to the average values, Model (a) and the black curve has been obtained using the average value of energy release rate and the maximum value of the Young modulus, Model (b).

The experimental results, under displacement control show a load drop in the critical load at the onset of propagation; this is due to an unstable propagation of the crack which grows catastrophically and arrests near the mid-span. The homogenized model, which is under crack-length control, is able to capture the snap-back instability and follow the virtual branch where crack growth is associated to a reduction of the load-point displacement. Crack propagation is modelled also in the region beyond the mid-span where the curve stably approaches the limiting solution (dotted line) corresponding to two fully delaminated layers.

\subsection{Layered beam (sandwich) with a single delamination, Fig. 4.b}

The material and geometrical properties of the three-layer (sandwich) bend beam in Fig. 4.b are given in Table 4. The layers are incompressible and isotropic with Dundurs' interface parameters $\alpha=0.6, \beta=0$ For this geometry and material properties, the delamination surfaces are in compression and the model is applicable assuming frictionless contact. The energy release rate is calculated numerically using the J-integral in Eq. (32) along the path shown in Fig. 3c, with $a$ and $c$ are chosen at sufficient distance from the crack tip and the relevant boundaries. The 2D solution is presented in Table 5 [48][55]. The third and fourth terms account for the effects of shear on the near tip deformations generated by the bending moments and the shear forces, respectively. The mode mixity phase angle, is $83.1^{\circ}$. Table 6 shows relative error between predictions made through the homogenized approach and the $2 \mathrm{D}$ solution. The results highlight the accuracy of the homogenized approach also for layered beams.

The diagram in Fig. 7 highlights the effects of the layered structure on the fracture properties. The dimensionless critical load for crack propagation, calculated using the model and $2 \mathrm{D}$ elasticity (Table 5) is shown versus normalized crack length in the sandwich beam of Tables 4 (red curves). The results are compared with those obtained for Beam 2 in Table 2, which is homogeneous with ${ }^{(2)} E /{ }^{(1)} E=1$ and a delamination at the same thickness-wise location. The effects of the layup on the critical load for crack propagation are important and well captured by the homogenized model. The 34 
reduced accuracy of the solutions for the homogeneous beam, already discussed in Section 4.1, depends mainly on the crack tip conditions which have an important Mode I component, $\psi=65.2^{\circ}$.

Table 4. Geometrical and material properties of the three-layer (sandwich) beam in Fig. 4.b

\begin{tabular}{|l|c|c|c|c|c|}
\hline & Thickness & Thickness ratios & \multicolumn{3}{|c|}{ Isotropic layers } \\
\hline & $H=3 h$ & ${ }^{(2)} h /{ }^{(1)} h,{ }^{(3)} h /{ }^{(1)} h$ & ${ }^{(2)} E /{ }^{(1)} E$ & ${ }^{(3)} E /{ }^{(1)} E$ & ${ }^{(1)} v,{ }^{(2)} v,{ }^{(3)} v$ \\
\hline Sandwich Beam & ${ }^{(1)} h+{ }^{(2)} h+{ }^{(3)} h$ & 1 & $1 / 4$ & 1 & 0.5 \\
\hline
\end{tabular}

Table 5-2D elasticity solution for the Energy Release Rate of the sandwich beam in Table 4, Fig. $4 \mathrm{~b}$.

\begin{tabular}{|l|l|c|}
\hline & \multicolumn{1}{|c|}{ 2D Elasticity } & Source \\
\hline Sandwich beam & $\frac{G^{(1)} \bar{E} h}{P^{2}}=0.265\left(\frac{a}{h}\right)^{2}\left[1-0.484\left(\frac{h}{a}\right)^{2}+0.739\left(\frac{h}{a}\right)+0.621\left(\frac{h}{a}\right)^{2}\right]$ & {$[48]$} \\
& $\psi=\tan ^{-1}\left(\sqrt{G_{I I} / G_{I}}\right)=83.1^{\circ}$ & \\
\hline
\end{tabular}

Table 6: Relative error on the energy release rate. Error is between results of the homogenized model and 2D solution.

\begin{tabular}{|l|c|c|c|c|c|c|}
\hline Relative error against 2D solution & Beam & $\frac{a}{h}=10$ & $\frac{a}{h}=15$ & $\frac{a}{h}=20$ & $\frac{a}{h}=30$ & $\frac{a}{h}=40$ \\
\hline J-integral, numerical, Eq. (32) & Sandwich Beam & $-7.3 \%$ & $-4.8 \%$ & $-3.6 \%$ & $-2.3 \%$ & $-1.7 \%$ \\
\hline $\begin{array}{l}\text { J-Integral (crack surface } \\
\text { displacement), numerical, Eq. (34) }\end{array}$ & Sandwich Beam & $-6.8 \%$ & $-4.6 \%$ & $-3.4 \%$ & $-2.3 \%$ & $-1.7 \%$ \\
\hline $\begin{array}{l}\text { Compliance method, numerical, } \\
\text { Eq. (31) }\end{array}$ & Sandwich Beam & $-8.9 \%$ & $-5.5 \%$ & $-4.0 \%$ & $-2.5 \%$ & $-1.8 \%$ \\
\hline
\end{tabular}

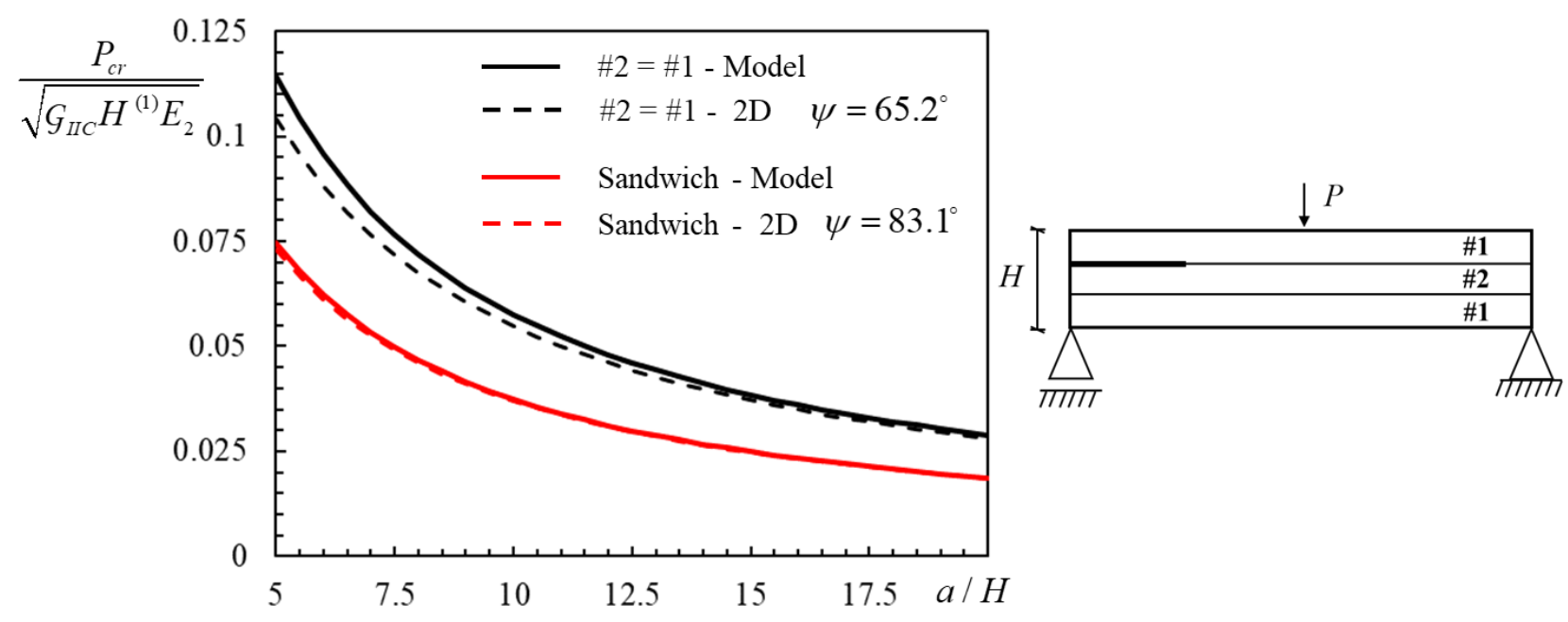

Fig. 7. Dimensionless diagram of critical load for crack propagation versus crack length in three-point bend beams with half-length $L>20 H$. Black curves: homogeneous beam, ${ }^{(2)} E /{ }^{(1)} E=1$; red curves: sandwich beam, ${ }^{(2)} E /{ }^{(1)} E=1 / 4$. 


\subsection{Cantilever beam with multiple delaminations, Fig. 4.c}

The cantilever beam in Fig. 4.c with two unequally spaced, unequal length delaminations is analyzed in this section. The interactions between delaminations induce phenomena of amplification or shielding of the energy release rate of a crack as it would be in the absence of other cracks; this behavior is controlled by length and spacing of the delaminations and has profound effects on the macrostructural behavior of laminated and sandwich beams [56][8][57]. In addition, when there are multiple delaminations extensive contact may occur along the crack faces [57,58][15]. The application is used to discuss capabilities and limitations of the homogenized model in the description of these phenomena. The beam is assumed to be homogeneous, isotropic and incompressible. Different geometries are examined and details are given in Table 7.

Table 7. Geometrical and material properties of the multiply delaminated cantilever beam in Fig. 4.c

\begin{tabular}{|c|c|c|c|c|c|c|c|c|}
\hline \multirow[t]{2}{*}{ Beam } & \multirow{2}{*}{$\begin{array}{c}\text { Thickness } \\
h\end{array}$} & \multirow{2}{*}{$\begin{array}{c}\text { Length } \\
L\end{array}$} & \multicolumn{2}{|c|}{ Crack Lengths } & \multicolumn{2}{|c|}{ Thickness ratios } & \multicolumn{2}{|c|}{$\begin{array}{c}\text { Isotropic } \\
\text { layers }\end{array}$} \\
\hline & & & $a_{U} / h$ & $a_{L} / h$ & ${ }^{(2)} h /{ }^{(1)} h$ & ${ }^{(3)} h /{ }^{(1)} h$ & ${ }^{(i)} E$ & ${ }^{(i)} v$ \\
\hline Cantilever Beam 1 & ${ }^{(1)} h+{ }^{(2)} h+{ }^{(3)} h$ & $10 h$ & varying & 5 & 1 & 1 & $E$ & 0.5 \\
\hline Cantilever Beam 2 & ${ }^{(1)} h+{ }^{(2)} h+{ }^{(3)} h$ & $10 h$ & $\begin{array}{c}\text { varying } \\
a_{U 0} / h=5.5\end{array}$ & $\begin{array}{c}\text { varying } \\
a_{U 0} / h=6\end{array}$ & 2 & 1 & $E$ & 0.5 \\
\hline Cantilever Beam 3 & ${ }^{(1)} h+{ }^{(2)} h+{ }^{(3)} h$ & $10 h$ & $\begin{array}{c}\text { varying } \\
a_{U 0} / h=5\end{array}$ & $\begin{array}{c}\text { varying } \\
a_{U 0} / h=4\end{array}$ & $1 / 3$ & $1 / 3$ & $E$ & 0.5 \\
\hline
\end{tabular}

Figure 8 shows the dimensionless energy release rate of the upper crack in the Cantilever Beam 1 of Table 7 on varying its length for a fixed length of the lower crack, $a_{L}=0.5 L$, calculated using the compliance method, Eq. (31), (thick solid lines). The results are compared with 2D Finite Element results in [8] (red triangles). The dashed curve defines the 2D solution of the upper crack in the absence of the lower crack [46] (single crack limit). The effect of crack interaction is an important amplification of the energy release rate with respect to the single crack limit. The effect is negligible only when $a_{U}>a_{L}$. The homogenized model accurately captures the interaction effects but when the difference in the length of the interacting delaminations is less than a few times the separation of their planes. In this region, the model predicts a discontinuity of the energy release rate, when $a_{U}=a_{L}$ , and overestimates (for $a_{U} \leq a_{L}$ ) or underestimates (for $a_{U} \geq a_{L}$ ) the 2D solution. The 2D solution in [46] shows that for $a_{U} \leq a_{L}$ the crack tip conditions are mode II, $\psi=\arctan \left(\sqrt{G_{I I} / G_{I}}\right)=90^{\circ}$, and 
contact between the crack faces occurs when $a_{L} \approx a_{U}$; for $a_{U}>a_{L}$, there is a sharp transition and the mode I component is suddenly increased to a phase angle $\psi \simeq 55^{\circ}$; on increasing the length of the upper crack, the phase angle then approaches that corresponding to the single crack limit, $\psi=66.7^{\circ}$

The results obtained with two discrete-layer models which assume constrained contact conditions (green dots) and elastic-contact along the crack surfaces (spring-contact model, dotted line) are also presented in the figure [8][12]. The constrained contact results coincide with the results of the homogenized model. The response substantially improves when contact between the crack surfaces is properly modelled. This comparison suggests a possible improvement of the homogenized model using the extended version of the multiscale model in [19], which may be used to account for crack opening and elastic contact.

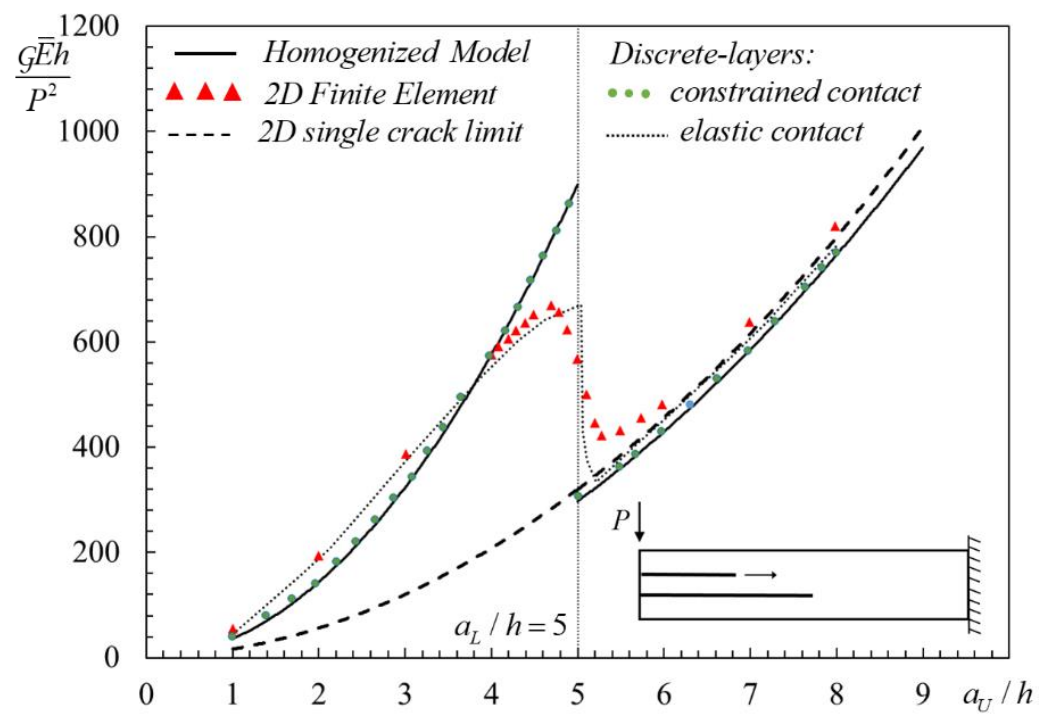

Fig. 8. Dimensionless diagram of the energy release rate of the upper crack in the Cantilever Beam 1 of Table 7, Fig. 4.c. Length of lower crack fixed at $a_{L} / h=5.2 \mathrm{D}$ and discrete-layer model results in $[8,12]$.

Figures 9 and 10 show the effects of the interaction on the macrostructural behavior. The dimensionless diagrams depict the critical load for the propagation of the cracks in the Cantilever Beams 2 and 3, in Table 7, versus load-point deflection. The delaminations are assumed to propagate when the energy release rate, calculated using the compliance method, Eq. (31), approaches the critical value, $G_{I I C}$. The results of the homogenized model are compared with the results of the discrete-layer cohesive-crack model with spring-contact in [12] and the same propagation criterion. A local snap-through instability is observed in the Cantilever Beam 2 when the upper crack starts to propagate in A and approaches the lower crack tip, in B. Then the load to propagate the crack must be increased, due to a shielding phenomenon, up to point $\mathrm{C}$ where the two cracks propagate together 
unstably. In the Cantilever Beam 3 the lower crack, which is shorter, starts to propagate at the maximum load, point A; crack propagation is unstable and characterized by a snap-back instability up to point $\mathrm{B}$. Then there is a sudden drop in the load, to point $\mathrm{C}$, caused by a sudden amplification discontinuity. After point $\mathrm{C}$ the lower crack continues its propagation.

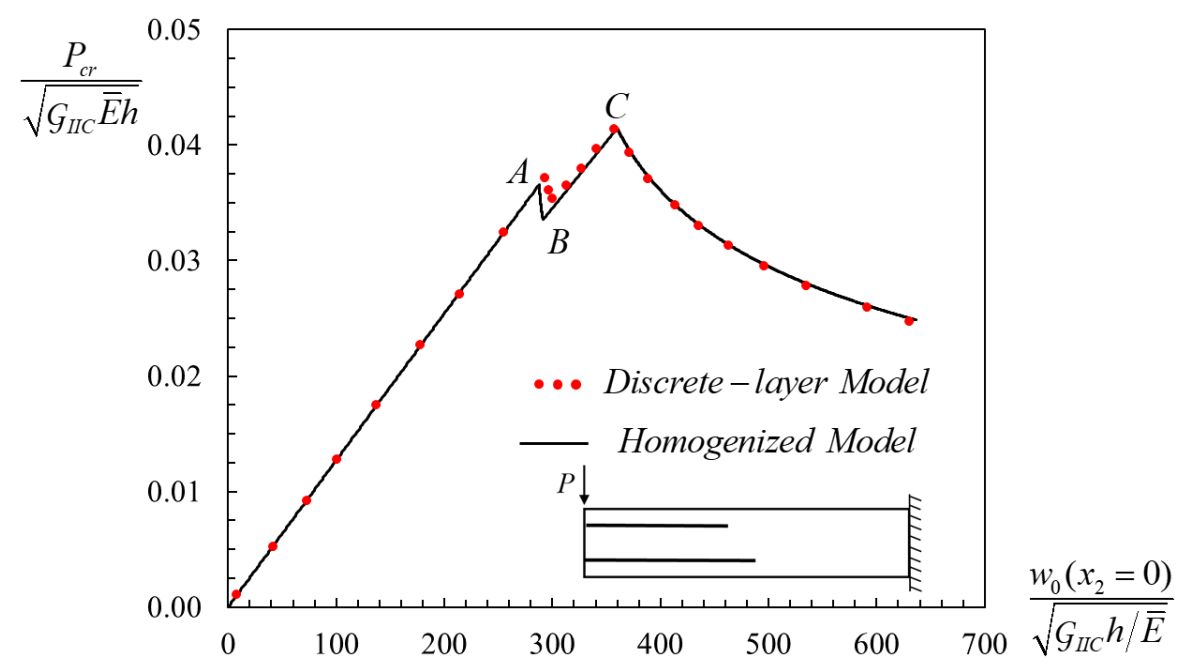

Fig. 9. Dimensionless diagram of the critical load for crack propagation versus load point displacement. Cantilever Beam 2 in Table 7, Fig. 4c. Discrete-layer model results in $[8,12]$.

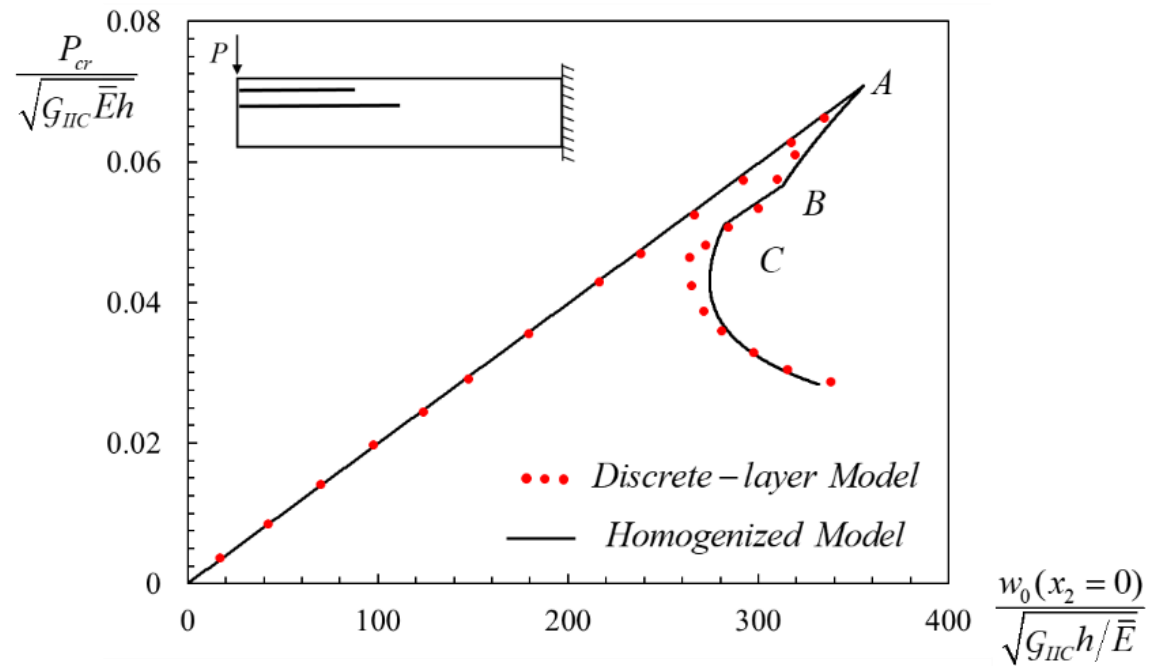

Fig. 10. Dimensionless diagram of the critical load for crack propagation in the Cantilever Beam 3 in Table 7 , Fig. 4.c. and discrete layer model results in $[8,12]$.

The diagrams in Figs. 8-10 highlight advantages and limitations of the homogenized approach. The model is able to capture, with only three displacement variables, complex interaction phenomena occurring between multiple delaminations and the qualitative behaviors are well captured. The results are less accurate when the difference in length of the delaminations is within a 
few times their spacing, mainly due to the assumptions of the model which neglects the transverse compressibility of the layers and contact along the crack surfaces.

\section{Conclusions}

Mode II dominant brittle fracture of homogeneous and layered beams is analyzed using a homogenized structural model based on a zigzag approach. The model captures the unstable propagation of cracks in bend-beams, snap-back and snap-through instabilities, the effects of the interaction of multiple cracks on the macrostructural response and the effects of the layered structure on the energy release rate and critical load for crack propagation.

The model is based on an extension of a classical zigzag theory, with the minimum number of unknowns, to beams and wide-plates with delaminations, which are described as cohesive interfaces and are included in the homogenized description. It applies to layers with principal material axes parallel to the geometrical axes, is controlled by three displacement variables, for any numbers of layers and delaminations, and rigorous fracture mechanics methods are used to derive the energy release rate and grow the cracks.

Closed form solutions have been derived for the displacement variables of general delaminated beams and for the energy release rate of homogeneous and bi-material beams with a single delamination. The energy release rate is in terms of crack tip force and moment sub-resultants and can be directly compared with accurate $2 \mathrm{D}$ solutions from the literature. The particularization to bi-material beams highlights the features of the model and the effects of the homogenization on the local fields in the layers and at the crack tip. The energy release rate in other geometries, namely layered beams and beams with multiple delaminations, is calculated using the compliance method and the J-Integral.

The limitations of the homogenized approach have been discussed. The first is a consequence of the imposed continuity of shear tractions and interfacial cohesive tractions at the layer interfaces; this implies that in the delaminated region of the plate the shear stresses and strains vanish and therefore their effects on the transverse compliance of the structure is neglected. Accurate shear stresses and strains can be calculated a posteriori from the bending stresses by imposing local equilibrium and their effect included in the energy release rate; and the resultant of these a-posteriori calculated stresses is variationally consistent. The second limitation is due to the imposition of continuity conditions on the global quantities. This implies that the near tip deformations, which are typically described using crack tip root rotations, are not accounted for in homogeneous beams and are only partially considered in bi-material beams thanks to the accurate description of the zigzag 
fields. A third limitation, which has been discussed in [44] and needs to be properly tackled if crack propagation has to be controlled directly from the cohesive tractions, as is it usually done in cohesivecrack modeling, regards the presence of a boundary region at the crack tip where the interfacial cohesive tractions are not accurately predicted by the model; this is again a consequence of the imposition of continuity conditions on the global variables.

Different assumptions and homogenization techniques could be used to limit the effects of neglecting the shear strains in the delaminated regions and to overcome the limitations of the zigzag theory used within this model ( $\mathrm{C}^{1}$ continuity; a posteriori definition of the shear stresses), typically at the expenses of an increased number of unknowns, [27]. This could be important for future implementation of the model into finite element codes. An attempt has been recently made in [38] using the refined zigzag theory in [25], which has been modified to include the cohesive interfaces. However, a preliminary investigation shows some inconsistencies when using that approach in the imposition of continuity conditions between regions with large variations of the homogenized properties, which is the case when dealing with plates with finite length delaminations. The same inconsistency has been noted in [37].

The investigation presented in this paper is limited to mode II dominant fracture of beams and wide-plates with a single delamination. The results are promising, and further work is necessary to verify the capabilities of this method to study single and multiple delaminations, under general mixed-mode conditions, cohesive fracture in Mode I, Mode II and Mode III and for the fracture analysis of more complex systems, such as plates and shells. The extension should be possible using the extended version of the homogenized model in $[19,20]$ or other homogenization techniques, with the difficulties already mentioned above.

Acknowledgements: The support by the U.S. Navy, Office of Naval Research, ONR, grants N00014-17-1-2914 monitored by Dr. Y.D.S. Rajapakse and by the Italian Department for University and Scientific and Technological Research in the framework of the research MIUR Prin15 project 2015LYYXA8 is gratefully acknowledged.

\section{References}

[1] Alfano G, Crisfield MA. Finite element interface models for the delamination analysis of laminated composites: Mechanical and computational issues. Int J Numer Methods Eng 2001. doi:10.1002/nme.93.

[2] Allix O, Ladevèze P. Interlaminar interface modelling for the prediction of delamination. Compos Struct 1992. doi:10.1016/0263-8223(92)90060-P.

[3] Allix O, Ladevéze P, Corigliano A. Damage analysis of interlaminar fracture specimens. Compos Struct 1995. doi:10.1016/0263-8223(95)00002-X.

[4] Mi Y, Crisfield MA, Davies GAO, Hellweg HB. Progressive delamination using interface elements. J Compos Mater 1998. doi:10.1177/002199839803201401.

[5] Borg R, Nilsson L, Simonsson K. Modeling of delamination using a discretized cohesive zone and damage 
formulation. Compos Sci Technol 2002. doi:10.1016/S0266-3538(02)00070-2.

[6] Turon A, Camanho PP, Costa J, Renart J. Accurate simulation of delamination growth under mixed-mode loading using cohesive elements: Definition of interlaminar strengths and elastic stiffness. Compos Struct 2010. doi:10.1016/j.compstruct.2010.01.012.

[7] Lundsgaard-Larsen C, Massabò R, Cox BN. On acquiring data for large-scale crack bridging at high strain rates. J Compos Mater 2012;46. doi:10.1177/0021998311413622.

[8] Andrews MG, Massabò R, Cox BN. Elastic interaction of multiple delaminations in plates subject to cylindrical bending. Int J Solids Struct 2006;43. doi:10.1016/j.ijsolstr.2005.04.025.

[9] Williams TO, Addessio FL. A general theory for laminated plates with delaminations. Int J Solids Struct 1997. doi:10.1016/S0020-7683(96)00131-X.

[10] Barbero EJ, Reddy JN. Modeling of delamination in composite laminates using a layer-wise plate theory. Int J Solids Struct 1991. doi:10.1016/0020-7683(91)90200-Y.

[11] Bruno D, Greco F. Mixed mode delamination in plates: A refined approach. Int J Solids Struct 2001. doi:10.1016/S0020-7683(01)00179-2.

[12] Andrews MG, Massabò R. Delamination in flat sheet geometries with material imperfections and thickness variations. Compos Part B Eng 2008;39. doi:10.1016/j.compositesb.2007.02.017.

[13] Szekrényes A. Nonsingular crack modelling in orthotropic plates by four equivalent single layers. Eur J Mech A/Solids 2016. doi:10.1016/j.euromechsol.2015.08.005.

[14] Szekrényes A. The role of transverse stretching in the delamination fracture of softcore sandwich plates. Appl Math Model 2018;63:611-32. doi:10.1016/j.apm.2018.07.014.

[15] Massabò R, Cavicchi A. Interaction effects of multiple damage mechanisms in composite sandwich beams subject to time dependent loading. Int J Solids Struct 2012;49. doi:10.1016/j.ijsolstr.2011.11.012.

[16] Rybicki EF, Kanninen MF. A finite element calculation of stress intensity factors by a modified crack closure integral. Eng Fract Mech 1977. doi:10.1016/0013-7944(77)90013-3.

[17] di Sciuva M. Bending, vibration and buckling of simply supported thick multilayered orthotropic plates: An evaluation of a new displacement model. J Sound Vib 1986. doi:10.1016/0022-460X(86)90169-0.

[18] Librescu L, Schmidt R. A general linear theory of laminated composite shells featuring interlaminar bonding imperfections. Int J Solids Struct 2001. doi:10.1016/S0020-7683(00)00263-8.

[19] Massabò R, Campi F. An efficient approach for multilayered beams and wide plates with imperfect interfaces and delaminations. Compos Struct 2014;116:311-24. doi:10.1016/j.compstruct.2014.04.009.

[20] Massabò R, Campi F. Assessment and correction of theories for multilayered plates with imperfect interfaces. Meccanica 2015;50. doi:10.1007/s11012-014-9994-x.

[21] Abrate S, Di sciuva M. 1.16 Multilayer Models for Composite and Sandwich Structures. Compr Compos Mater II 2018:399-425.

[22] Carrera E. Historical review of Zig-Zag theories for multilayered plates and shells. Appl Mech Rev 2003. doi:10.1115/1.1557614.

[23] Averill RC. Static and dynamic response of moderately thick laminated beams with damage. Compos Eng 1994. doi:10.1016/S0961-9526(09)80013-0.

[24] Averill RC, Yip YC. Development of simple, robust finite elements based on refined theories for thick laminated beams. Comput Struct 1996. doi:10.1016/0045-7949(95)00269-3.

[25] Tessler A, Di Sciuva M, Gherlone M. A refined zigzag beam theory for composite and sandwich beams. J. Compos. Mater., 2009. doi:10.1177/0021998308097730.

[26] Tessler A, Di Sciuva M, Gherlone M. A consistent refinement of first-order shear deformation theory for laminated composite and sandwich plates using improved zigzag kinematics. J Mech Mater Struct 2010. doi:10.2140/jomms.2010.5.341.

[27] Tessler A. Refined zigzag theory for homogeneous, laminated composite, and sandwich beams derived from Reissner's mixed variational principle. Meccanica 2015;50:2621-48. doi:10.1007/s11012-015-0222-0.

[28] Iurlaro L, Gherlone M, Di Sciuva M, Tessler A. Refined Zigzag Theory for laminated composite and sandwich plates derived from Reissner's Mixed Variational Theorem. Compos Struct 2015;133:809-17. doi:10.1016/j.compstruct.2015.08.004.

[29] Pelassa M, Massabò R. Explicit solutions for multi-layered wide plates and beams with perfect and imperfect bonding and delaminations under thermo-mechanical loading. Meccanica 2015;50. doi:10.1007/s11012-0150147-7.

[30] Massabò R. Propagation of Rayleigh-Lamb waves in multilayered plates through a multiscale structural model. Int J Solids Struct 2017;124. doi:10.1016/j.ijsolstr.2017.06.020.

[31] Massabò R. Influence of boundary conditions on the response of multilayered plates with cohesive interfaces and delaminations using a homogenized approach. Frat Ed Integrita Strutt 2014;8. doi:10.3221/IGF-ESIS.29.20.

[32] Icardi U, Zardo G. COplate element for delamination damage analysis, based on a zig-zag model and strain energy updating. Int J Impact Eng 2005. doi:10.1016/j.ijimpeng.2004.02.002.

[33] Groh RMJ, Tessler A. Computationally efficient beam elements for accurate stresses in sandwich laminates and laminated composites with delaminations. Comput Methods Appl Mech Eng 2017. 
doi:10.1016/j.cma.2017.03.035.

[34] Eijo A, Oñate E, Oller S. Delamination in laminated plates using the 4-noded quadrilateral QLRZ plate element based on the refined zigzag theory. Compos Struct 2014. doi:10.1016/j.compstruct.2013.09.052.

[35] Eijo A, Oñate E, Oller S. A numerical model of delamination in composite laminated beams using the LRZ beam element based on the refined zigzag theory. Compos Struct 2013. doi:10.1016/j.compstruct.2013.04.035.

[36] Groh RMJ, Weaver PM, Tessler A. Application of the Refined Zigzag Theory to the Modeling of Delaminations in Laminated Composites. 2015. doi:10.13140/RG.2.1.3147.0804.

[37] Flores FG, Oller S, Nallim LG. On the analysis of non-homogeneous laminates using the refined zigzag theory. Compos Struct 2018. doi:10.1016/j.compstruct.2018.08.018.

[38] Darban, H., Massabò R. A homogenized structural model for shear deformable composites with compliant interlayers. Multiscale Multidiscip Model Exp Des 2018;in press.

[39] Banks-Sills L, Arcan M, Bortman Y. A mixed mode fracture specimen for mode II dominant deformation. Eng Fract Mech 1984. doi:10.1016/0013-7944(84)90122-X.

[40] Martin RH, Davidson BD. Mode II fracture toughness evaluation using four point bend, end notched flexure test. Plast Rubber Compos 1999. doi:10.1016/j.spl.2017.11.006.

[41] Tao J, Sun CT. Influence of ply orientation on delamination in composite laminates. J Compos Mater 1998. doi:10.1177/002199839803202103.

[42] Yang PC, Norris CH, Stavsky Y. Elastic wave propagation in heterogeneous plates. Int J Solids Struct 1966. doi:10.1016/0020-7683(66)90045-X.

[43] Whitney JM, Sun CT. A higher order theory for extensional motion of laminated composites. J Sound Vib 1973. doi:10.1016/S0022-460X(73)80052-5.

[44] Darban H. Multiscale modeling of delamination fracture in multilayered structures. University of Genova, Italy, 2018.

[45] Brandinelli L, Massabò R. Mode II weight functions for isotropic and orthotropic double cantilever beams. Int J Fract 2006;139. doi:10.1007/s10704-006-6358-0.

[46] Li S, Wang J, Thouless MD. The effects of shear on delamination in layered materials. J Mech Phys Solids 2004. doi:10.1016/S0022-5096(03)00070-X.

[47] Thouless MD. The effects of transverse shear on the delamination of edge-notch flexure and 3-point bend geometries. Compos Part B Eng 2009. doi:10.1016/j.compositesb.2009.01.005.

[48] Barbieri L, Massabò R, Berggreen C. The effects of shear and near tip deformations on interface fracture of symmetric sandwich beams. Eng Fract Mech 2018:1-24. doi:10.1016/j.engfracmech.2018.06.039.

[49] Andrews MG, Massabò R. The effects of shear and near tip deformations on energy release rate and mode mixity of edge-cracked orthotropic layers. Eng Fract Mech 2007;74. doi:10.1016/j.engfracmech.2007.01.013.

[50] Fraisse P, Schmit F. Use of J-integral as fracture parameter in simplified analysis of bonded joints. Int J Fract 1993. doi:10.1007/BF00053316.

[51] Thouless MD. Shear forces, root rotations, phase angles and delamination of layered materials. Eng Fract Mech 2018. doi:10.1016/j.engfracmech.2018.01.033.

[52] L.A., Carlsson; Adams, D.F.; Pipes RB. Experimental Characterization of advanced composite materials. IV Edition. CRC Press; n.d.

[53] Madhukar MS, Drzal LT. Fiber-Matrix Adhesion and Its Effect on Composite Mechanical Properties: IV. Mode I and Mode II Fracture Toughness of Graphite/Epoxy Composites. J Compos Mater 1992;26:936-68. doi:10.1177/002199839202600701.

[54] Madhukar MS, Drzal LT. Fiber-Matrix Adhesion and Its Effect on Composite Mechanical Properties: II. Longitudinal $\left(0^{\circ}\right)$ and Transverse $\left(90^{\circ}\right)$ Tensile and Flexure Behavior of Graphite/Epoxy Composites. J Compos Mater 1991;25:958-91. doi:10.1177/002199839102500802.

[55] Østergaard RC, Sørensen BF. Interface crack in sandwich specimen. Int J Fract 2007. doi:10.1007/s10704-0079059-4.

[56] Zheng S, Sun CT. Delamination interaction in laminated structures. Eng Fract Mech 1998. doi:10.1016/S00137944(97)00120-3.

[57] Suemasu H. Postbuckling Behaviors of Composite Panels with Multiple Delaminations. J Compos Mater 1993. doi:10.1177/002199839302701102.

[58] Larsson PL. On multiple delamination buckling and growth in composite plates. Int J Solids Struct 1991. doi:10.1016/0020-7683(91)90065-N.

\section{Appendix A - Coefficients and boundary conditions}

Coefficients of the homogenized constitutive and equilibrium equations of the layered beam: 


$$
\begin{array}{ll}
C^{r S}=\sum_{k=1}^{n}{ }^{(k)} \bar{E}_{2} \int_{x_{3}^{k-1}}^{x_{3}^{k}}\left(x_{3}\right)^{r} R_{S 22}^{k} d x_{3} & C^{S 2}=\sum_{k=1}^{n}{ }^{(k)} \bar{E}_{2} \int_{x_{3}^{k-1}}^{x_{3}^{k}}\left(R_{S 22}^{k}\right)^{2} d x_{3} \\
\left.C_{44}^{P}=\sum_{k=1}^{n}{ }^{(k)} G_{23} \int_{x_{3}^{k-1}}^{x_{3}^{k}}\left(1+R_{S 22}^{k}\right)_{3}\right)^{2} d x_{3} & C^{S}=K_{S}\left(\Psi_{22}\right)^{2}
\end{array}
$$

Homogenized boundary conditions, Eq. (13), in terms of displacement variables:

$$
\begin{aligned}
& \left(A_{22} v_{02},_{2}+B_{22} \varphi_{2,2}+C^{0 S} w_{0, r_{22}}\right) n_{2}=\tilde{N}_{2} \quad \text { or } \quad v_{02}=\tilde{v}_{02} \\
& {\left[B_{22} v_{02},_{2}+\left(D_{22}+C^{1 S}\right) \varphi_{2,2}+C^{1 S} w_{0},{ }_{22}\right] n_{2}=\tilde{M}_{2}^{b} \text { or } \varphi_{2}=\tilde{\varphi}_{2}} \\
& {\left[A_{44}\left(\varphi_{2}+w_{0}, 2\right)-C^{0 S} v_{02,,_{22}}-\left(C^{1 S}+C^{S 2}\right) \varphi_{2},_{22}-C^{S 2} w_{0},{ }_{222}\right] n_{2}=\tilde{N}_{3} \text { or } w_{0}=\tilde{w}_{0}} \\
& \left(C^{0 S} v_{02,2}+\left(C^{1 S}+C^{S 2}\right) \varphi_{2,2}+C^{S 2} w_{0,22}\right) n_{2}=\tilde{M}_{2}^{z S} \text { or } \quad w_{0},_{2}=\tilde{w}_{0}, 2
\end{aligned}
$$

Coefficients of the general solution, Eq. (19), for a bi-material or $n$-layer wide-plate/beam:

$$
\begin{aligned}
& \bar{A}=\frac{C^{0 S} B_{22}-C^{1 S} A_{22}}{B_{22}\left(B_{22}+C^{0 S}\right)-\left[D_{22}+C^{1 S}\right] A_{22}} \\
& \bar{B}=\frac{A_{22}(1-\bar{A}) A_{44}}{\bar{A}\left[\left(B_{22}+C^{0 S}\right)^{2}-\left(D_{22}+2 C^{1 S}+C^{S 2}\right) A_{22}\right]-\left(B_{22}+C^{0 S}\right) C^{0 S}+\left(C^{1 S}+C^{S 2}\right) A_{22}} \\
& \bar{C}=\frac{\bar{B}}{A_{44}(\bar{A}-1)} \quad ; \quad \bar{D}=\frac{\left(B_{22}+C^{0 S}\right)^{2}-D_{22} A_{22}}{B_{22}\left(B_{22}+C^{0 S}\right)-\left[D_{22}+C^{1 S}\right] A_{22}} \\
& \bar{E}=\frac{A_{44} A_{22}}{B_{22}\left(B_{22}+C^{0 S}\right)-\left[D_{22}+C^{1 S}\right] A_{22}}
\end{aligned}
$$

